\title{
Effective neutrino masses in KATRIN and future tritium beta-decay experiments
}

\author{
Guo-yuan Huang, ${ }^{1,2, *}$ Werner Rodejohann $\oplus^{3, \dagger}$ and Shun Zhou ${ }^{1,2, \hbar}$ \\ ${ }^{1}$ Institute of High Energy Physics, Chinese Academy of Sciences, Beijing 100049, China \\ ${ }^{2}$ School of Physical Sciences, University of Chinese Academy of Sciences, Beijing 100049, China \\ ${ }^{3}$ Max-Planck-Institut für Kernphysik, Postfach 103980, D-69029 Heidelberg, Germany
}

(Received 25 October 2019; published 3 January 2020)

\begin{abstract}
Past and current direct neutrino mass experiments set limits on the so-called effective neutrino mass, which is an incoherent sum of neutrino masses and lepton mixing matrix elements. The electron energy spectrum which neglects the relativistic and nuclear recoil effects is often assumed. Alternative definitions of effective masses exist, and an exact relativistic spectrum is calculable. We quantitatively compare the validity of those different approximations as function of energy resolution and exposure in view of tritium beta decays in the KATRIN, Project 8, and PTOLEMY experiments. Furthermore, adopting the Bayesian approach, we present the posterior distributions of the effective neutrino mass by including current experimental information from neutrino oscillations, beta decay, neutrinoless double-beta decay, and cosmological observations. Both linear and logarithmic priors for the smallest neutrino mass are assumed.
\end{abstract}

DOI: 10.1103/PhysRevD.101.016003

\section{INTRODUCTION}

Neutrino oscillation experiments have measured with very good precision the three leptonic flavor mixing angles $\left\{\theta_{12}, \theta_{13}, \theta_{23}\right\}$ and two independent neutrino mass-squared differences $\Delta m_{21}^{2} \equiv m_{2}^{2}-m_{1}^{2}$ and $\left|\Delta m_{31}^{2}\right| \equiv$ $\left|m_{3}^{2}-m_{1}^{2}\right|$. The absolute scale of neutrino masses, however, has to be determined from nonoscillation approaches, using beta decay [1], neutrinoless doublebeta decay [2], or cosmological observations [3]. Once the neutrino mass scale is established, one knows the lightest neutrino mass, which is $m_{1}$ in the case of normal neutrino mass ordering (NO) with $m_{1}<m_{2}<m_{3}$, or $m_{3}$ in the case of inverted neutrino mass ordering (IO) with $m_{3}<m_{1}<m_{2}$.

As first suggested by Fermi and Perrin [4-6], the precise measurement of the electron energy spectrum in nuclear beta decays ${ }_{Z}^{A} N \rightarrow \underset{Z+1}{A} N+e^{-}+\bar{\nu}_{e}$, where $A$ and $Z$ denote the mass and the atomic number of the decaying nucleus, can be utilized to probe absolute neutrino masses. Since the energy released in beta decays is distributed to massive neutrinos, the energy spectrum of electrons in the

\footnotetext{
*huanggy@ihep.ac.cn

werner.rodejohann@mpi-hd.mpg.de

zhoush@ihep.ac.cn
}

Published by the American Physical Society under the terms of the Creative Commons Attribution 4.0 International license. Further distribution of this work must maintain attribution to the author(s) and the published article's title, journal citation, and DOI. Funded by SCOAP ${ }^{3}$. region close to its end point will be distorted in comparison to that in the limit of zero neutrino masses. This kinematic effect is usually described by the effective neutrino mass [7]

$$
m_{\beta} \equiv \sqrt{\left|U_{e 1}\right|^{2} m_{1}^{2}+\left|U_{e 2}\right|^{2} m_{2}^{2}+\left|U_{e 3}\right|^{2} m_{3}^{2}},
$$

where $U_{e i}$ (for $i=1,2,3$ ) stand for the first-row elements of the leptonic flavor mixing matrix $U$, i.e., $\left|U_{e 1}\right|=$ $\cos \theta_{13} \cos \theta_{12},\left|U_{e 2}\right|=\cos \theta_{13} \sin \theta_{12},\left|U_{e 3}\right|=\sin \theta_{13}$ in the standard parametrization [8], and $m_{i}$ (for $i=1,2,3$ ) for the absolute neutrino masses. Very recently, the KATRIN Collaboration has reported its first result on the effective neutrino mass using tritium beta decay ${ }^{3} \mathrm{H} \rightarrow{ }^{3} \mathrm{He}+e^{-}+\bar{\nu}_{e}$ and reached the currently most stringent upper bound $[9,10]$

$$
m_{\beta}<1.1 \mathrm{eV}
$$

at the $90 \%$ confidence level (CL). With the full exposure in the near future, KATRIN aims for an ultimate limit of $m_{\beta}<0.2 \mathrm{eV}$ at the same CL [11], which is an order of magnitude better than the result $m_{\beta} \lesssim 2 \mathrm{eV}$ from the Mainz [12] and Troitsk [13] experiments.

Motivated by this impressive achievement of the KATRIN experiment, we revisit the validity of the effective neutrino mass $m_{\beta}$ in Eq. (1) and clarify how it depends on the energy resolution and the sensitivity of a realistic 
TABLE I. The configurations of tritium beta-decay experiments and the resulting $\chi_{\beta}^{2}$ and $\Delta \chi_{\text {true }}^{2}$ defined in Eqs. (19) and (20) arising from the description of the electron spectrum by using the effective neutrino mass $m_{\beta}$. One year of data taking has been assumed. No background is assumed, and the $\chi^{2}$ values can be further reduced taking into account possible background contributions.

\begin{tabular}{|c|c|c|c|c|c|c|}
\hline$m_{\mathrm{L}}=0 \mathrm{eV}$ & Target mass & $\Delta$ & $\chi_{\beta}^{2}, \mathrm{NO}$ & $\chi_{\beta}^{2}, \mathrm{IO}$ & $\Delta \chi_{\text {true }}^{2}, \mathrm{NO}$ & $\Delta \chi_{\text {true }}^{2}, \mathrm{IO}$ \\
\hline KATRIN & $2.5 \times 10^{-4} \mathrm{~g}$ & $1 \mathrm{eV}$ & $7.4 \times 10^{-7}$ & $5.6 \times 10^{-7}$ & $1.3 \times 10^{-7}$ & $1.1 \times 10^{-7}$ \\
\hline Project 8 (Molecular $\left.{ }^{3} \mathrm{H}\right)$ & $5 \times 10^{-4} \mathrm{~g}$ & $0.36 \mathrm{eV}$ & $8.9 \times 10^{-5}$ & $6.1 \times 10^{-5}$ & $2.0 \times 10^{-5}$ & $1.4 \times 10^{-5}$ \\
\hline Project 8 (Atomic ${ }^{3} \mathrm{H}$ ) & $5 \times 10^{-4} \mathrm{~g}$ & $0.05 \mathrm{eV}$ & 0.064 & 0.13 & 0.032 & 0.015 \\
\hline PTOLEMY & $100 \mathrm{~g}$ & $0.15 \mathrm{eV}$ & 428 & 331 & 141 & 81 \\
\hline
\end{tabular}

experiment for tritium beta decays. ${ }^{1}$ More explicitly, we shall consider the KATRIN [11], Project 8 [17], and PTOLEMY [18-20] experiments. Their main features and projected sensitivities have been summarized in Appendix A and Table I. We compare $m_{\beta}$ in Eq. (1) with other effective neutrino masses proposed in the literature and also consider the exact relativistic spectrum of tritium beta decays. A measure of the validity of $m_{\beta}$ in terms of the exposure and energy resolution for a beta-decay experiment can be set. As a result, we find that the standard effective mass $m_{\beta}$ and the classical spectrum form can be used for KATRIN and Project 8 essentially without losing accuracy.

Furthermore, it is of interest to estimate how likely a signal in upcoming neutrino mass experiments, including those using electron capture, is. Toward this end, we perform a Bayesian analysis to obtain the posterior distributions of $m_{\beta}$. The probability to find the beta-decay signal depends on the experimental likelihood input one considers, in particular the neutrino mass information from cosmology and neutrinoless double-beta decays. The cosmological constraints on neutrino masses reply on the datasets one has included in generating the likelihood, whereas the constraints from neutrinoless double-beta decays are subject to the assumption whether neutrinos are Dirac or Majorana particles. It is thus quantified what the consequences of adding more and more additional mass information are. Moreover, the prior on the smallest neutrino mass, which could be linear or logarithmic, is important for the final posteriors.

The remaining part of our paper is organized as follows. In Sec. II, we make a comparison between the exact relativistic spectrum of electrons from tritium beta decays with the ordinary one with an effective neutrino mass $m_{\beta}$. Then, a quantitative assessment of the validity of the effective neutrino mass is carried out. The posterior distributions of the effective neutrino mass are calculated in Sec. III, where the present experimental information from neutrino oscillations, neutrinoless double-beta decays, and cosmology are included. Finally, we summarize our

\footnotetext{
${ }^{1}$ In this work, we focus only on tritium beta-decay experiments. Similar analyses of the effective neutrino mass can be performed for the electron-capture decays of holmium, namely, $e^{-}+{ }^{163} \mathrm{Ho} \rightarrow \nu_{e}+{ }^{163} \mathrm{Dy}$, which are and will be investigated in the ECHo [14] and HOLMES [15], NuMECS [16] experiments.
}

main results in Sec. IV. Technical details on the considered experiments and on the likelihoods used for our Bayesian analysis are delegated to Appendices.

\section{THE EFFECTIVE NEUTRINO MASS}

\section{A. The relativistic electron spectrum}

Before introducing the effective neutrino mass for beta decays, we present the exact relativistic energy spectrum of the outgoing electrons for tritium beta decays (or equivalently the differential decay rate), which can be calculated within standard electroweak theory [21-24], the result being

$$
\frac{\mathrm{d} \Gamma_{\text {rel }}}{\mathrm{d} K_{e}}=N_{\mathrm{T}} \frac{\bar{\sigma}\left(E_{e}\right)}{\pi^{2}} \sum_{i=1}^{3}\left|U_{e i}\right|^{2} H\left(E_{e}, m_{i}\right) .
$$

Here $N_{\mathrm{T}}$ is the target mass of ${ }^{3} \mathrm{H}$, and $E_{e}=K_{e}+m_{e}$ is the electron energy with $K_{e}$ being its kinetic energy. In Eq. (3), the reduced cross section is given by

$$
\begin{aligned}
\bar{\sigma}\left(E_{e}\right) \equiv & \frac{G_{\mathrm{F}}^{2}}{2 \pi}\left|V_{\mathrm{ud}}\right|^{2} F\left(Z, E_{e}\right) \frac{m^{3 \mathrm{He}}}{m_{3 \mathrm{H}}} E_{e} \sqrt{E_{e}^{2}-m_{e}^{2}} \\
& \times\left[\left\langle f_{\mathrm{F}}\right\rangle^{2}+\left(\frac{C_{\mathrm{A}}}{C_{\mathrm{V}}}\right)^{2}\left\langle g_{\mathrm{GT}}\right\rangle^{2}\right],
\end{aligned}
$$

where $G_{\mathrm{F}}=1.166 \times 10^{-5} \mathrm{GeV}^{-2}$ is the Fermi constant, $\left|V_{\mathrm{ud}}\right| \approx \cos \theta_{\mathrm{C}}$ is determined by the Cabibbo angle $\theta_{\mathrm{C}} \approx 12.8^{\circ}, F\left(Z, E_{e}\right)$ is the ordinary Fermi function with $Z=1$ for tritium taking account of the distortion of the electron wave function in the Coulomb potential of the decaying nucleus, ${ }^{2} C_{\mathrm{V}} \approx 1$ and $C_{\mathrm{A}} \approx 1.2695$ stand for the vector and axial-vector coupling constants of the charged-current weak interaction of nucleons, respectively. In addition, $\left\langle f_{\mathrm{F}}\right\rangle^{2} \approx 0.9987$ and $\left\langle g_{\mathrm{GT}}\right\rangle^{2} \approx 2.788$ are the squared nuclear matrix elements of the allowed Fermi and Gamow-Teller transitions. The kinematics of the tritium beta decays is encoded in the function $H\left(E_{e}, m_{i}\right)$ in Eq. (3), namely,

\footnotetext{
${ }^{2}$ The Fermi function is given by $F\left(Z, E_{e}\right)=2 \pi \eta /\left(1-e^{-2 \pi \eta}\right)$, where $\eta \equiv Z \alpha E_{e} / p_{e}$ with $p_{e}=\sqrt{E_{e}^{2}-m_{e}^{2}}$ being the electron momentum and $\alpha \approx 1 / 137$ the fine-structure constant.
} 


$$
\begin{aligned}
H\left(E_{e}, m_{i}\right) \equiv & \frac{1-m_{e}^{2} /\left(m_{3_{\mathrm{H}}} E_{e}\right)}{\left(1-2 E_{e} / m_{3_{\mathrm{H}}}+m_{e}^{2} / m_{3_{\mathrm{H}}}^{2}\right)^{2}} \\
& \times \sqrt{y\left(y+\frac{2 m_{i} m_{3^{\mathrm{He}}}}{m_{{ }^{3} \mathrm{H}}}\right)}\left[y+\frac{m_{i}}{m_{{ }^{\mathrm{H}}}}\left(m_{{ }^{\mathrm{He}}}+m_{i}\right)\right],
\end{aligned}
$$

where $\quad y \equiv K_{\text {end }}-K_{e} \quad$ with $\quad K_{\text {end }}=\left[\left(m_{3_{\mathrm{H}}}-m_{e}\right)^{2}-\right.$ $\left.\left(m_{3 \mathrm{He}}+m_{i}\right)^{2}\right] /\left(2 m_{3 \mathrm{H}}\right)$ being the end point energy corresponding to the neutrino mass $m_{i}$. Some comments on the kinematics are in order:

(i) Given the nuclear masses $m_{3} \mathrm{H} \approx 2808920.8205 \mathrm{keV}$ and $m_{3 \mathrm{He}} \approx 2808391.2193 \mathrm{keV}$ [23], as well as the electron mass $m_{e} \approx 510.9989 \mathrm{keV}$, one can obtain the $Q$ value of tritium beta decay $Q \equiv$ $m_{{ }^{3} \mathrm{H}}-m_{3_{3} \mathrm{He}}-m_{e} \approx 18.6023 \mathrm{keV}$. In the limit of vanishing neutrino masses, the end point energy $K_{\text {end }}$ turns out to be

$$
\begin{aligned}
K_{\mathrm{end}, 0} & \equiv\left[\left(m_{3^{\mathrm{H}}}-m_{e}\right)^{2}-m_{3_{\mathrm{He}}}^{2}\right] /\left(2 m_{3^{\mathrm{H}}}\right) \\
& \approx 18.5989 \mathrm{keV},
\end{aligned}
$$

which is lower than the $Q$ value by a small amount of $Q-K_{\text {end, } 0} \approx 3.4 \mathrm{eV}$. This difference arises from the recoil energies of the final-state particles and is naturally included when one considers fully relativistic kinematics. Since the electron spectrum near its end point is sensitive to absolute neutrino masses, which are much smaller than this energy difference of $3.4 \mathrm{eV}$, it is not appropriate to treat the $Q$ value as the end point energy.

(ii) It is straightforward to verify that $K_{\text {end }} \approx K_{\text {end, } 0}-$ $m_{i} m_{3^{\mathrm{He}}} / m_{3_{\mathrm{H}}}$ and thus $y+m_{i} m_{3^{\mathrm{He}}} / m_{3^{\mathrm{H}}} \approx K_{\text {end, },}-$ $K_{e}$, where a tiny term $m_{i}^{2} /\left(2 m_{3_{3}}\right)<1.78 \times$ $10^{-10} \mathrm{eV}$ for $m_{i}<1 \mathrm{eV}$ can be safely ignored. Taking this approximation on the right-hand side of Eq. (5), we can recast the kinematical function into

$$
\begin{aligned}
H\left(E_{e}, m_{i}\right) \approx & \frac{1-m_{e}^{2} /\left(m_{3_{\mathrm{H}}} E_{e}\right)}{\left(1-2 E_{e} / m_{{ }^{\mathrm{H}}}+m_{e}^{2} / m_{{ }^{\mathrm{H}}}^{2}\right)^{2}} \\
& \times \sqrt{\left(K_{\text {end }, 0}-K_{e}\right)^{2}-\left(m_{i} \frac{m_{{ } \mathrm{He}}}{m_{{ }^{\mathrm{H}}}}\right)^{2}} \\
& \times\left(K_{\text {end }, 0}-K_{e}\right),
\end{aligned}
$$

from which it is interesting to observe that the absolute neutrino mass $m_{i}$ in the square root receives a correction factor $m_{3 \mathrm{He}} / m_{3 \mathrm{H}} \approx 0.999811$. The difference between Eqs. (7) and (5) is negligibly small, so the former will be used in the following discussions.
Furthermore, given $1-m^{3} \mathrm{He} / m_{3} \mathrm{H} \approx 1.89 \times 10^{-4}$ and $m_{e} / m^{3} \mathrm{H} \approx 1.82 \times 10^{-4}$, the relativistic electron spectrum $\mathrm{d} \Gamma_{\text {rel }} / \mathrm{d} K_{e}$ approximates to the classical one

$$
\begin{aligned}
\frac{\mathrm{d} \Gamma_{\mathrm{c} 1}}{\mathrm{~d} K_{e}}= & N_{\mathrm{T}} \frac{\bar{\sigma}_{\mathrm{cl}}\left(E_{e}\right)}{\pi^{2}} \sum_{i=1}^{3}\left|U_{e i}\right|^{2} \sqrt{\left(K_{\text {end }, 0}-K_{e}\right)^{2}-m_{i}^{2}} \\
& \times\left(K_{\text {end }, 0}-K_{e}\right),
\end{aligned}
$$

where $\bar{\sigma}_{\mathrm{cl}}\left(E_{e}\right)=\bar{\sigma}\left(E_{e}\right) /\left(m_{{ }^{3} \mathrm{He}} / m_{3_{\mathrm{H}}}\right)$ and $\bar{\sigma}\left(E_{e}\right)$ has been given in Eq. (4). Comparing the classical spectrum in Eq. (8) with the relativistic one in Eq. (3), one can observe that the end point energy in the former case deviates from the true one by an amount of $\left(1-m_{3_{\mathrm{He}}} / m_{3_{\mathrm{H}}}\right) m_{i} \approx 10^{-4} m_{i}$. As the PTOLEMY experiment could achieve a relative precision of $10^{-6}$ for the determination of the lightest neutrino mass [20], it would be no longer appropriate to use the classical spectrum in PTOLEMY. However, it is rather safe for KATRIN and Project 8 to neglect the factor $1-m^{3} \mathrm{He} / m_{3 \mathrm{H}}$, as their sensitivities to the neutrino mass are weaker than for PTOLEMY. To be more specific, the $1 \sigma$ sensitivities of KATRIN and Project 8 to $m_{\beta}^{2}$ are $\sigma\left(m_{\beta}^{2}\right) \approx$ $0.025 \mathrm{eV}^{2}$ [11] and $\sigma\left(m_{\beta}^{2}\right) \approx 0.001 \mathrm{eV}^{2}$ [17], respectively, corresponding to $\sigma\left(m_{\beta}\right) / m_{\beta} \approx 0.05\left(0.5 \mathrm{eV} / m_{\beta}\right)^{2}$ and $\sigma\left(m_{\beta}\right) / m_{\beta} \approx 0.002\left(0.5 \mathrm{eV} / m_{\beta}\right)^{2}$. Both values are much larger than the correction of order $10^{-4}$ from the factor $m_{3^{3} \mathrm{He}} / m_{3_{3} \mathrm{H}}$. To have an expression for the electron spectrum applicable to experiments beyond KATRIN and Project 8, i.e., leading to PTOLEMY, we can slightly modify the classical energy spectrum as follows:

$$
\begin{aligned}
\frac{\mathrm{d} \Gamma_{\mathrm{cl}}^{\prime}}{\mathrm{d} K_{e}}= & N_{\mathrm{T}} \frac{\bar{\sigma}_{\mathrm{cl}}\left(E_{e}\right)}{\pi^{2}} \sum_{i=1}^{3}\left|U_{e i}\right|^{2} \sqrt{\left(K_{\mathrm{end}, 0}-K_{e}\right)^{2}-\left(m_{i} \frac{m_{3_{\mathrm{He}}}}{m_{{ }^{\mathrm{H}}}}\right)^{2}} \\
& \times\left(K_{\mathrm{end}, 0}-K_{e}\right) .
\end{aligned}
$$

Let us now check whether the difference between the exact relativistic spectrum $\mathrm{d} \Gamma_{\text {rel }} / \mathrm{d} K_{e}$ and the modified classical one $\mathrm{d} \Gamma_{\mathrm{cl}}^{\prime} / \mathrm{d} K_{e}$ affects the determination of absolute neutrino masses in future beta-decay experiments with a target mass of tritium ranging from $10^{-4} \mathrm{~g}$ in KATRIN to $100 \mathrm{~g}$ in PTOLEMY. In other words, we examine whether these two spectra are statistically distinguishable in realistic experiments. Consider the ratio of these two energy spectra

$$
\begin{aligned}
\frac{\mathrm{d} \Gamma_{\text {rel }} / \mathrm{d} K_{e}}{\mathrm{~d} \Gamma_{\mathrm{cl}}^{\prime} / \mathrm{d} K_{e}} & =\frac{1-m_{e}^{2} /\left(m_{{ }^{H}} E_{e}\right)}{\left(1-2 E_{e} / m_{{ }^{3} \mathrm{H}}+m_{e}^{2} / m_{{ }^{\mathrm{H}}}^{2}\right)^{2}} \cdot \frac{m_{3_{\mathrm{He}}}}{m_{{ }^{\mathrm{H}}}} \\
& \approx 1.0036+1.7 \times 10^{-9}\left(\frac{K_{e}}{\mathrm{eV}}\right),
\end{aligned}
$$

where an expansion in terms of the electron kinetic energy $K_{e}=E_{e}-m_{e}$ has been carried out. First of all, the constant on the rightmost side of Eq. (10) can be 
absorbed into the uncertainty of the overall normalization factor $A_{\beta}$ in the statistical analysis, so it is irrelevant for our discussions. ${ }^{3}$ Considering the term proportional to the electron kinetic energy $K_{e}$ in Eq. (10), it could potentially disturb the determination of the normalization factor of the spectrum. The distortion amplitude induced by the $K_{e^{-}}$ dependent term can be characterized by the specified energy window $\Delta K_{e}$ below the end point. For example, we have $\Delta K_{e}=30 \mathrm{eV}$ for KATRIN while $\Delta K_{e}=5 \mathrm{eV}$ for PTOLEMY, which is limited by the detector performance; see Appendix A. In this way, we can obtain the distortion amplitude of $5.1 \times 10^{-8}$ for KATRIN and $8.5 \times 10^{-9}$ for PTOLEMY, respectively. To examine the impact of this distortion, one can compare it with the statistical fluctuation of the events within the corresponding energy window. The integrated number of beta-decay events within the energy window below the end point can be calculated via

$$
\begin{aligned}
N_{\text {int }} & =T \int_{K_{\text {end, }, 0}-\Delta K_{e}}^{K_{\text {end, } 0}} \frac{\mathrm{d} \Gamma}{\mathrm{d} K_{e}} \mathrm{~d} K_{e} \\
& \approx 3.2 \times 10^{11} \cdot\left(\frac{\Delta K_{e}}{\mathrm{eV}}\right)^{3} \cdot\left(\frac{\mathcal{E}}{100 \mathrm{~g} \cdot \mathrm{yr}}\right),
\end{aligned}
$$

where $T$ is the operation time and $\mathcal{E} \equiv N_{\mathrm{T}} \cdot T$ is the total exposure. The statistical fluctuation of the beta-decay events within the energy window is estimated as $\sqrt{N_{\text {int }}} / N_{\text {int }} \approx 10^{-5}$ for KATRIN with $\Delta K_{e}=30 \mathrm{eV}$ and $\mathcal{E}=10^{-4} \mathrm{~g} \cdot \mathrm{yr}$, while $\sqrt{N_{\text {int }}} / N_{\text {int }} \approx 10^{-7}$ for PTOLEMY with $\Delta K_{e}=5 \mathrm{eV}$ and $\mathcal{E}=100 \mathrm{~g} \cdot \mathrm{yr}$. Both values are much larger than the corresponding distortion amplitudes. It is thus evident that the uncertainty in $A_{\beta}$ will be dominated by the intrinsic statistical fluctuation of the observed beta-decay events in future experiments. As the data fluctuation near the end point is most significant among the entire spectrum, the influence of the spectral distortion as indicated in Eq. (10) is not important. Hence, we conclude that the classical spectrum with the neutrino masses corrected by $m_{i} \rightarrow m_{i} \cdot\left(m_{3 \mathrm{He}} / m_{3 \mathrm{H}}\right)$ in Eq. (9) works as well as the exact relativistic spectrum in Eq. (3) for future beta-decay experiments.

It is worthwhile to emphasize that because of a finite energy resolution $\Delta$, which is normally much larger than the absolute neutrino mass $m_{i}$, it is difficult to resolve the true end point. Hence, the experimental sensitivity to neutrino masses is in fact governed by the integrated number of beta-decay events within a specified energy window below the end point. Taking this energy window to be the experimental energy resolution, $\Delta K_{e}=\Delta$, we can

\footnotetext{
${ }^{3}$ For instance, in the statistical analysis of the simulated data for the PTOLEMY experiment [20], the prior of the normalization factor $A_{\beta}$ is set to be in the range of $(0 \ldots 2)$, which is wide enough to take account of the difference corresponding to the constant term in the ratio in Eq. (10).
}

figure out the expected statistics around the end point according to Eq. (11). For a conservative experimental setup, e.g., that is achievable in KATRIN with $\Delta=1 \mathrm{eV}$ and $\mathcal{E}=10^{-4} \mathrm{~g} \cdot \mathrm{yr}$, the expected event number within the end point bin is $3.2 \times 10^{5}$. In the limit of $m_{\beta} \ll \Delta$, a finite neutrino mass will induce a relative deviation of events within the window $\Delta$ by $3 / 2 \cdot m_{\beta}^{2} / \Delta^{2}$. Therefore, the sensitivity to $m_{\beta}$ is roughly scaled as $(\Delta / \mathcal{E})^{1 / 4}$. The choice of energy window is limited by the smearing effect of finite energy resolution, and the sensitivity to neutrino masses will drop with a larger energy resolution. This can be compensated by increasing the exposure, such that an efficient event number can be acquired to resolve the overall shift due to finite neutrino masses.

Unless stated otherwise, we will refer from now on to $\mathrm{d} \Gamma_{\mathrm{cl}}^{\prime} / \mathrm{d} K_{e}$ as the exact spectrum in the remaining discussion.

\section{B. Validity of the effective mass}

In principle, it is the exact relativistic spectrum that should be confronted with the experimental observation in order to extract the absolute neutrino masses $m_{i}$ (for $i=1$, 2, 3), since $\left|U_{e i}\right|^{2}$ (for $i=1,2,3$ ) can be precisely measured in neutrino oscillation experiments. However, often the effective electron spectrum with only one mass parameter is considered (see e.g., [25]),

$$
\begin{aligned}
\frac{\mathrm{d} \Gamma_{\mathrm{eff}}}{\mathrm{d} K_{e}}= & N_{\mathrm{T}} \frac{\bar{\sigma}_{\mathrm{c} 1}\left(E_{e}\right)}{\pi^{2}} \sqrt{\left(K_{\mathrm{end}, 0}-K_{e}\right)^{2}-\left(m_{\beta} \frac{m_{3 \mathrm{He}}}{m_{3 \mathrm{H}}}\right)^{2}} \\
& \times\left(K_{\text {end }, 0}-K_{e}\right),
\end{aligned}
$$

where the effective neutrino mass $m_{\beta}$ is usually defined as in Eq. (1). Note that for consistency we have kept the nearunity factor $m_{{ }^{3} \mathrm{He}} / m_{3_{\mathrm{H}}}$ as in $\mathrm{d} \Gamma_{\mathrm{cl}}^{\prime} / \mathrm{d} K_{e}$ of Eq. (9), which is necessary when it comes to experiments beyond KATRIN and Project 8, i.e., PTOLEMY. However, $m^{{ }^{3} \mathrm{He}} / m^{3} \mathrm{H}$ appears as an overall factor to all neutrino mass parameters, so the quantitative impact on our discussion of the validity of the effective mass is actually negligible, ${ }^{4}$ but we keep it nevertheless in our numerical calculations. Another important point is that the end point energy in Eq. (6) should be corrected if we take account of binding energies as well as excitations of the daughter system in an actual experiment. For instance, in KATRIN or Project 8 with the molecular tritium target, a correction of $16.29 \mathrm{eV}$ to the end point energy should be considered owing to the binding energies

\footnotetext{
${ }^{4}$ One can easily check that the relation $m_{\beta}^{2} \equiv\left|U_{e 1}\right|^{2} m_{1}^{2}+$ $\left|U_{e 2}\right|^{2} m_{2}^{2}+\left|U_{e 3}\right|^{2} m_{3}^{2}$ is stable under $m_{i} \rightarrow m_{i} \cdot\left(m_{3 \mathrm{He}} / m_{3_{\mathrm{H}}}\right)$ and $m_{\beta} \rightarrow m_{\beta} \cdot\left(m_{3^{H e}} / m_{3} \mathrm{H}\right)$. Thus, any quantitative conclusion made by considering $m_{3} \mathrm{He} / m_{3} \mathrm{H}$ corrections can be directly applied to the case without correction of $m_{3}{ }_{\mathrm{He}} / m_{3} \mathrm{H}$ by shifting all neutrino masses with a relative fraction as small as $10^{-4}$, and vice versa.
} 
of the mother tritium pair, the daughter tritium-helium molecule, and the combination of the ionized electron [1]. Compared to the atomic case, the recoil energy of the molecular state will also be reduced by a factor of two due to the doubled mass, which will boost the end point energy of electrons. For PTOLEMY, with a foreseen possibility of atomic tritium weakly bounded to a graphene layer [18], the ionized electron will inevitably interact with the complex graphene binding system. Furthermore, the recoiled helium (with a kinetic energy of $3.4 \mathrm{eV}$ ) will escape the graphene binding structure with a sub-eV binding energy. All these effects need to be systemically considered in the experiment to evaluate the final end point energy $K_{\text {end, } 0}$. However, in our case, the spectrum in Eq. (12) mostly depends on the relative deviation from the end point energy $K_{\text {end, } 0}-K_{e}$, and $\bar{\sigma}_{\mathrm{c} 1}\left(E_{e}\right)$ changes very slowly as a function of $K_{\text {end, } 0}-K_{e}$ near the end point. Hence, our results which will be presented in terms of $K_{\text {end, } 0}-K_{e}$ are still valid if a different $K_{\text {end, } 0}$ is considered. On the other hand, the final-state excitations of molecules will smear the energy of outgoing electrons [26-29], and this effect will be taken into account as the irreducible energy resolution of the experiment.

Let us summarize the existing expressions of the electron spectra defined in this work: (i) the exact relativistic beta spectrum $\mathrm{d} \Gamma_{\text {rel }} / \mathrm{d} K_{e}$ without making approximations; see Eq. (3); (ii) the classical spectrum $\mathrm{d} \Gamma_{\mathrm{cl}} / \mathrm{d} K_{e}$ in the limit of $m_{3 \mathrm{He}} / m^{3 \mathrm{H}} \rightarrow 1$ and $m_{e} / m_{3_{\mathrm{H}}} \rightarrow 0$; see Eq. (8); (iii) the modified classical spectrum $\mathrm{d} \Gamma_{\mathrm{cl}}^{\prime} / \mathrm{d} K_{e}$ by making the replacement $m_{i} \rightarrow m_{i} \cdot\left(m_{3^{3} \mathrm{He}} / m_{{ }^{3} \mathrm{H}}\right)$ in $\mathrm{d} \Gamma_{\mathrm{cl}} / \mathrm{d} K_{e} ;$ see Eq. (9); (iv) the effective electron spectrum $\mathrm{d} \Gamma_{\text {eff }} / \mathrm{d} K_{e}$ defined in Eq. (12). We have seen that the difference between $\mathrm{d} \Gamma_{\mathrm{cl}}^{\prime} / \mathrm{d} K_{e}$ in Eq. (9) and the classical spectrum $\mathrm{d} \Gamma_{\mathrm{cl}} / \mathrm{d} K_{e}$ in Eq. (8) plays only a role when PTOLEMY is considered. In addition, the difference to the relativistic spectrum $\mathrm{d} \Gamma_{\text {rel }} / \mathrm{d} K_{e}$ in Eq. (3) is minuscule and the classical spectra can be considered as the exact ones. It remains to compare the so-defined exact spectrum $\mathrm{d} \Gamma_{\mathrm{cl}}^{\prime} / \mathrm{d} K_{e}$ in Eq. (9) to the effective one $\mathrm{d} \Gamma_{\text {eff }} / \mathrm{d} K_{e}$ in Eq. (12).

Moreover, in the literature, two different definitions of the effective neutrino mass have also been introduced [30-32], namely,

$$
m_{\beta}^{\prime} \equiv \sum_{i=1}^{3} m_{i}\left|U_{e i}\right|^{2}, \quad m_{\beta}^{\prime \prime} \equiv m_{1} .
$$

The effective electron spectrum can then be obtained by replacing $m_{\beta}$ in Eq. (12) with $m_{\beta}^{\prime}$ or $m_{\beta}^{\prime \prime}$. In this subsection, we discuss the difference among those three effective neutrino masses and clarify their validity with future beta-decay experiments in mind.

As has been observed in Ref. [32], the three effective neutrino masses have different accuracies in fitting the exact spectrum. If neutrino masses are quasidegenerate, all three effective masses provide very good fits and their relative differences are very small. For example, it is easy to verify that $\left(m_{\beta}^{\prime 2}-m_{\beta}^{\prime \prime 2}\right) / m_{\beta}^{\prime \prime} \lesssim 10^{-3}$. If the chosen energy window satisfies $\Delta K_{e}<2 m_{\beta}$, then $m_{\beta}^{\prime}$ can give a better fit than $m_{\beta}^{\prime \prime}$, whereas $m_{\beta}$ is still an excellent parameter in fitting the spectrum with an almost negligible difference $\left(m_{\beta}^{2}-m_{\beta}^{\prime 2}\right) / m_{\beta}^{\prime \prime 2} \lesssim 10^{-5}$. If neutrino masses are hierarchical, $m_{\beta}$ always fits better to the spectrum than the other two variants. In case of an extremely small value of the lightest neutrino mass, both $m_{\beta}^{\prime}$ and $m_{\beta}^{\prime \prime}$ are unable to offer a good fit to the true spectrum. In Fig. 1, we plot three effective neutrino masses in terms of the lightest neutrino mass which is $m_{1}$ for NO and $m_{3}$ for IO. One can observe that their differences are significant in $\mathrm{NO}$ when $m_{1}$ is small, but in IO the differences are always unnoticeable. The situation of IO can be attributed to the fact that the contribution of $m_{3}$ is suppressed by $\left|U_{e 3}\right|^{2}$ while the remaining two neutrino masses $m_{1}$ and $m_{2}$ are always nearly degenerate due to the relation $\Delta m_{21}^{2} \ll\left|\Delta m_{31}^{2}\right|$.

To be more explicit, we look carefully at the main difference between the exact spectrum $\mathrm{d} \Gamma_{\mathrm{cl}}^{\prime} / \mathrm{d} K_{e}$ and the effective one $\mathrm{d} \Gamma_{\text {eff }} / \mathrm{d} K_{e}$. The difference stems from the kinematical functions, namely [32],

$$
\begin{aligned}
\mathrm{d} \Gamma_{\mathrm{cl}}^{\prime} / \mathrm{d} K_{e} \propto & \sum_{i=1}^{3}\left|U_{e i}\right|^{2} \sqrt{\left(K_{\mathrm{end}, 0}-K_{e}\right)^{2}-\left(m_{i} \cdot \frac{m_{{ }^{3} \mathrm{He}}}{m_{{ }^{\mathrm{H}}}}\right)^{2}} \\
& \times\left(K_{\text {end }, 0}-K_{e}\right)
\end{aligned}
$$

$$
\begin{aligned}
\mathrm{d} \Gamma_{\text {eff }} / \mathrm{d} K_{e} \propto & \sqrt{\left(K_{\text {end }, 0}-K_{e}\right)^{2}-\left(m_{\beta}^{(\prime, \prime \prime)} \cdot \frac{m_{3} \mathrm{He}}{m_{3 \mathrm{H}}}\right)^{2}} \\
& \times\left(K_{\text {end }, 0}-K_{e}\right),
\end{aligned}
$$

where the spectra involving our three effective neutrino masses $m_{\beta}, m_{\beta}^{\prime}$, and $m_{\beta}^{\prime \prime}$ are collectively given. To analyze the difference, we take $m_{\beta}$ in the NO case, for example, but the other effective masses and the IO case can be studied in a similar way. Let us start with the end point of the electron spectrum and then go to lower energies. For convenience, the factor of $m_{3} \mathrm{He} / m_{3} \mathrm{H}$ is omitted in the following qualitative discussion, which of course will not affect the main feature of the result as we noted above.

(1) For the exact spectrum, the end point energy $K_{\text {end }}$ is set by the smallest neutrino mass, i.e., $K_{\text {end, } 0}-K_{\text {end }}=m_{1}$, while it is $m_{\beta}$ for the effective spectrum $\mathrm{d} \Gamma_{\text {eff }} / \mathrm{d} K_{e}$. Since $m_{\beta}^{2}=m_{1}^{2}+\Delta m_{21}^{2}\left|U_{e 2}\right|^{2}+$ $\Delta m_{31}^{2}\left|U_{e 3}\right|^{2}>m_{1}^{2}$, the end point energy of the effective spectrum $\mathrm{d} \Gamma_{\text {eff }} / \mathrm{d} K_{e}$ is smaller than that of the exact one $\mathrm{d} \Gamma_{\mathrm{cl}}^{\prime} / \mathrm{d} K_{e}$. Therefore, starting from the electron kinetic energy of $K_{e}=K_{\text {end, } 0}-m_{1}$ and going to smaller values, the effective spectrum 

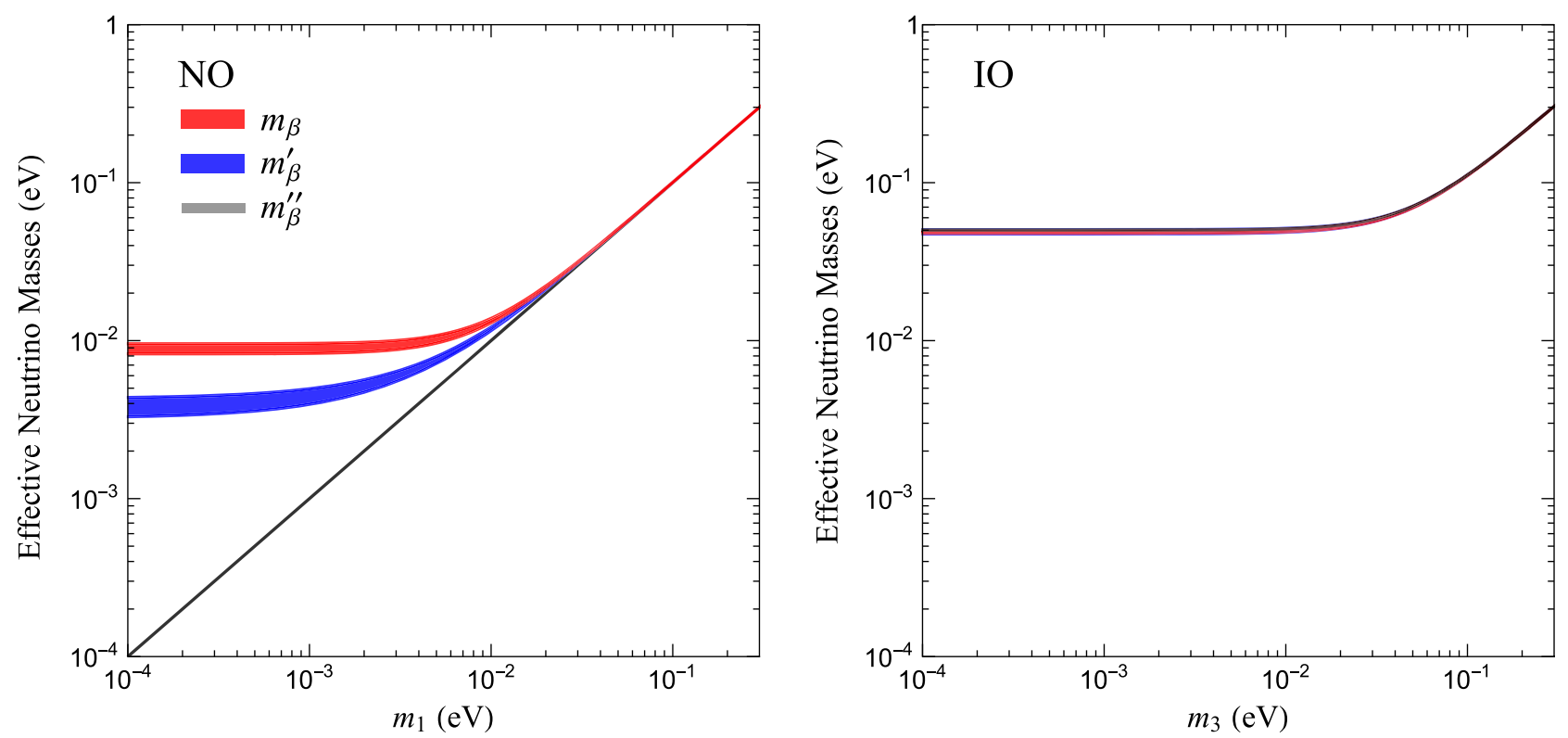

FIG. 1. The effective neutrino masses versus $m_{1}$ for NO (left panel) and $m_{3}$ for IO (right panel). The $3 \sigma$ CL uncertainties of oscillation parameters have been considered. A similar plot for absolute neutrino masses was given in Ref. [33].

$\mathrm{d} \Gamma_{\text {eff }} / \mathrm{d} K_{e}$ is always vanishing and thus should be lying below the exact one $\mathrm{d} \Gamma_{\mathrm{cl}}^{\prime} / \mathrm{d} K_{e}$.

(2) As $K_{e}$ is decreasing further, we come to the point at which $K_{\text {end }, 0}-K_{e}=m_{\beta}$ is satisfied. Note that $m_{\beta}^{2}=$ $m_{3}^{2}-\Delta m_{31}^{2}\left|U_{e 1}\right|^{2}-\Delta m_{32}^{2}\left|U_{e 2}\right|^{2}<m_{3}^{2}$ holds. Therefore, for $m_{\beta}<K_{\text {end, } 0}-K_{e}<m_{3}, \mathrm{~d} \Gamma_{\text {eff }} / \mathrm{d} K_{e}$ becomes nonzero. As indicated in Eqs. (14) and (15), before the decay channel corresponding to $m_{3}$ is switched on, $\mathrm{d} \Gamma_{\text {eff }} / \mathrm{d} K_{e}$ is about to exceed $\mathrm{d} \Gamma_{\mathrm{cl}}^{\prime} / \mathrm{d} K_{e}$. At $K_{e}=K_{\text {end }, 0}-m_{3}$, we have $\mathrm{d} \Gamma_{\text {eff }} / \mathrm{d} K_{e} \propto$ $\sqrt{\left|U_{e 1}\right|^{2} \Delta m_{31}^{2}+\left|U_{e 2}\right|^{2} \Delta m_{32}^{2}} \approx \sqrt{\left|U_{e 1}\right|^{2}+\left|U_{e 2}\right|^{2}}$ $\sqrt{\Delta m_{31}^{2}} \quad$ and $\quad \mathrm{d} \Gamma_{\mathrm{cl}}^{\prime} / \mathrm{d} K_{e} \propto\left|U_{e 1}\right|^{2} \sqrt{\Delta m_{31}^{2}}+$ $\left|U_{e 2}\right|^{2} \sqrt{\Delta m_{32}^{2}} \approx\left(\left|U_{e 1}\right|^{2}+\left|U_{e 2}\right|^{2}\right) \sqrt{\Delta m_{31}^{2}}$, where $\Delta m_{21}^{2} \ll \Delta m_{31}^{2}$ has been taken into account, leading to $\mathrm{d} \Gamma_{\text {eff }} / \mathrm{d} K_{e}>\mathrm{d} \Gamma_{\mathrm{cl}}^{\prime} / \mathrm{d} K_{e}$.

(3) When we go far below the end point, e.g., $K_{e} \ll$ $K_{\text {end }, 0}-m_{i}$ or equivalently $K_{\text {end, } 0}-K_{e} \gg m_{i}$, the neutrino masses can be neglected and thus these two spectra coincide with each other. Therefore, for $m_{\beta}$ under consideration, the difference between $\mathrm{d} \Gamma_{\text {eff }} / \mathrm{d} K_{e}$ and $\mathrm{d} \Gamma_{\mathrm{cl}}^{\prime} / \mathrm{d} K_{e}$ could change its sign in the narrow range below the end point but finally converges to zero.

For illustration, we show in Fig. 2 the electron spectra in the narrow energy region $-200 \mathrm{meV} \leq K_{e}-K_{\text {end, } 0} \leq$ $200 \mathrm{meV}$ around the end point, where possible background events are ignored. In addition, the total exposure for the tritium beta-decay experiment is taken to be $\mathcal{E}=1 \mathrm{~g} \cdot \mathrm{yr}$. In the left panel of Fig. 2, the exact spectrum $\mathrm{d} \Gamma_{\mathrm{cl}}^{\prime} / \mathrm{d} K_{e}$ with $m_{1}=10 \mathrm{meV}$ is plotted as the gray solid curve, while that with $m_{1}=10.5 \mathrm{meV}$ is represented by the red dotted curve for comparison. The effective spectra $\mathrm{d} \Gamma_{\text {eff }} / \mathrm{d} K_{e}$ for $m_{\beta}=13.4 \mathrm{meV}, m_{\beta}^{\prime}=11.9 \mathrm{meV}$, and $m_{\beta}^{\prime \prime}=$ $10 \mathrm{meV}$ are given by the dark, medium, and light blue dashed curves, respectively. Those values are obtained for a smallest mass of $m_{1}=10 \mathrm{meV}$ and the current best-fit values of the oscillation parameters [34]. Since it is hard to distinguish these spectra, as can be seen in the upper subgraph in the left panel, we depict their deviations from the exact spectrum,

$$
\Delta\left(\mathrm{d} \Gamma / \mathrm{d} K_{e}\right) \equiv \mathrm{d} \Gamma_{\text {eff }} / \mathrm{d} K_{e}-\mathrm{d} \Gamma_{\mathrm{cl}}^{\prime} / \mathrm{d} K_{e},
$$

with $m_{1}=10 \mathrm{meV}$ in the lower subgraph. The behavior of these deviations can be well understood analytically, as we have already explained by using Eqs. (14) and (15). As for the exact spectrum $\mathrm{d} \Gamma_{\mathrm{cl}}^{\prime} / \mathrm{d} K_{e}$ with $m_{1}=10.5 \mathrm{meV}$, it is always lying below that with $m_{1}=10 \mathrm{meV}$. The reason is simply that the kinematical function $\left[\left(K_{\text {end, } 0}-K_{e}\right)^{2}-\left(m_{i}\right.\right.$. $\left.\left.m_{3 \mathrm{He}} / m_{3 \mathrm{H}}\right)^{2}\right]^{1 / 2}$ in the exact spectrum $\mathrm{d} \Gamma_{\mathrm{cl}}^{\prime} / \mathrm{d} K_{e}$ becomes smaller for larger values of $m_{i}$.

The finite energy resolution of the detector has been ignored in the left panel of Fig. 2, but is taken into account in the calculations of the energy spectra and their deviations from $\mathrm{d} \Gamma_{\mathrm{cl}}^{\prime} / \mathrm{d} K_{e}$ with $m_{1}=10 \mathrm{meV}$ in the right panel. Assuming the energy resolution ${ }^{5}$ of the detector to be $\Delta=100 \mathrm{meV}$ and taking the Gaussian form, we can derive the energy spectrum with smearing effects as follows:

\footnotetext{
${ }^{5}$ The values of energy resolution in this work are all referred to as the $1 \sigma$ deviation of the energy reconstruction.
} 

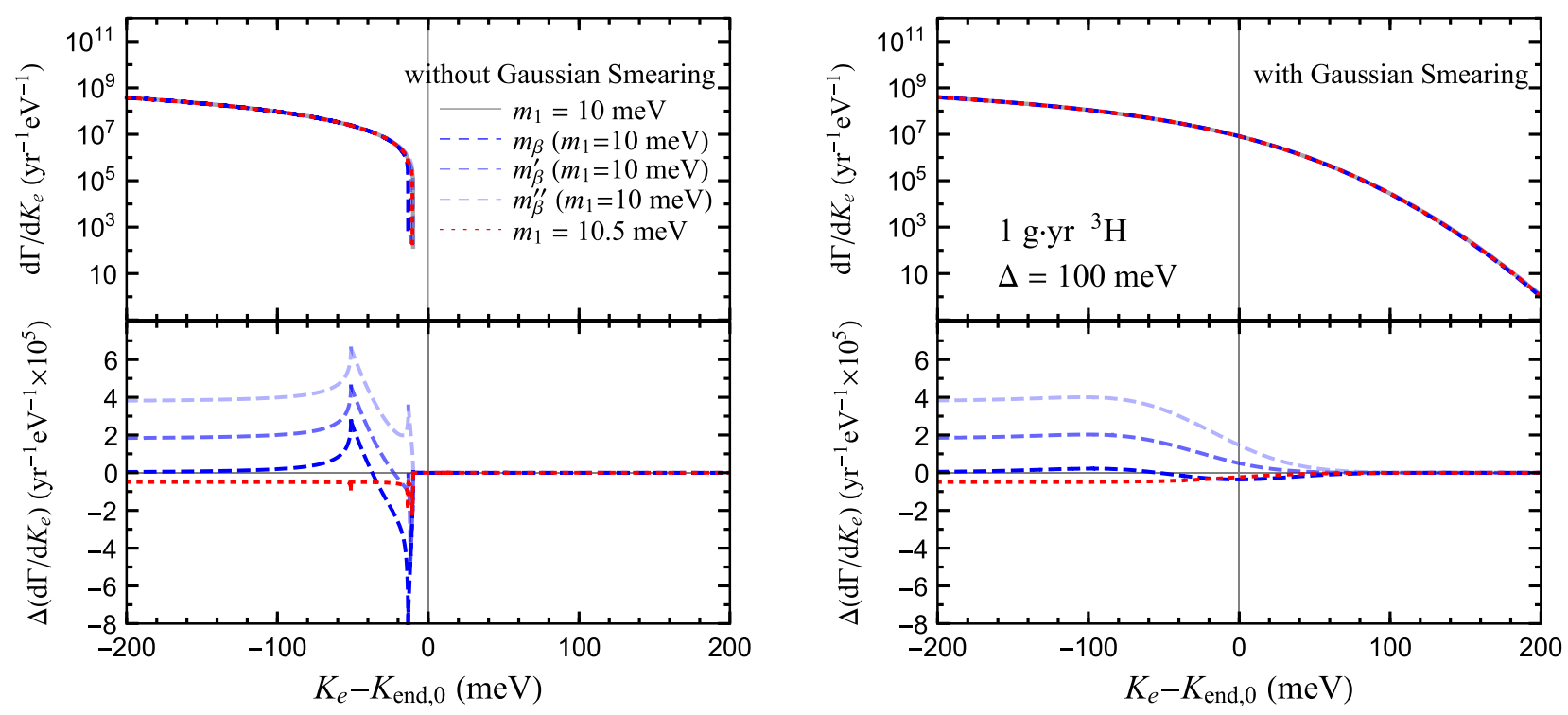

FIG. 2. Illustration of the electron spectrum from tritium beta decays, where the total exposure $\mathcal{E}=1 \mathrm{~g} \cdot \mathrm{yr}$ and the best-fit values of neutrino mixing angles and mass-squared differences are assumed. The exact spectra $\mathrm{d} \Gamma_{\mathrm{cl}}^{\prime} / \mathrm{d} K_{e}$ with $m_{1}=10 \mathrm{meV}$ and $m_{1}=10.5 \mathrm{meV}$ are shown as the gray solid and red dotted curve, respectively. The effective spectra $\mathrm{d} \Gamma_{\text {eff }} / \mathrm{d} K_{e}$ with $m_{\beta}=13.4 \mathrm{meV}, m_{\beta}^{\prime}=11.9 \mathrm{meV}$, and $m_{\beta}^{\prime \prime}=10 \mathrm{meV}$, corresponding to $m_{1}=10 \mathrm{meV}$, are represented by the dark, medium, and light blue dashed curves. In the left panel, the energy smearing is ignored, while an energy resolution of $\Delta=100 \mathrm{meV}$ is taken into account in the right panel. In both panels, the real spectra are depicted in the upper subgraph, whereas their deviations from the exact spectrum with $m_{1}=10$ meV are given in the lower subgraph.

$$
\begin{aligned}
\frac{\mathrm{d} \Gamma_{\Delta}}{\mathrm{d} K_{e}}\left(K_{e}\right)= & \frac{1}{\sqrt{2 \pi}(\Delta / \sqrt{8 \ln 2})} \int_{-\infty}^{+\infty} \mathrm{d} K_{e}^{\prime} \frac{\mathrm{d} \Gamma}{\mathrm{d} K_{e}^{\prime}}\left(K_{e}^{\prime}\right) \\
& \times \exp \left[-\frac{\left(K_{e}-K_{e}^{\prime}\right)^{2}}{2(\Delta \sqrt{8 \ln 2})^{2}}\right],
\end{aligned}
$$

which has been plotted in the right panel for both $\mathrm{d} \Gamma_{\mathrm{cl}}^{\prime} / \mathrm{d} K_{e}$ and $\mathrm{d} \Gamma_{\text {eff }} / \mathrm{d} K_{e}$. Note that we have not yet specified any planned experimental configuration so far, because the main purpose here is to understand the behavior of deviations caused by using different effective neutrino masses. Two interesting observations can be made and deserve further discussions.

(i) First, when energy smearing effects are included, the difference between $\mathrm{d} \Gamma_{\text {eff }} / \mathrm{d} K_{e}$ and $\mathrm{d} \Gamma_{\mathrm{cl}}^{\prime} / \mathrm{d} K_{e}$ will be averaged over the electron kinetic energy, reducing the discrepancy between them. This effect is more significant for the electron kinetic energy closer to the end point. Therefore, if the energy resolution is extremely good, the error caused by using the effective spectrum becomes larger. In this case, one needs to fit the experimental data by implementing $\mathrm{d} \Gamma_{\mathrm{cl}}^{\prime} / \mathrm{d} K_{e}$ with the lightest neutrino mass as the fundamental parameter.

(ii) Second, the effective spectrum $\mathrm{d} \Gamma_{\text {eff }} / \mathrm{d} K_{e}$ with $m_{\beta}$ converges to the exact one in the energy region far below the end point. Moreover, it is interesting to notice that even though the difference $\Delta\left(\mathrm{d} \Gamma / \mathrm{d} K_{e}\right)$ between $\mathrm{d} \Gamma_{\text {eff }} / \mathrm{d} K_{e}$ with $m_{\beta}$ and $\mathrm{d} \Gamma_{\text {cl }}^{\prime} / \mathrm{d} K_{e}$ can be either positive or negative, the total number of betadecay events within a very wide energy window is approximately vanishing. To be more concrete, the integration of $\Delta\left(\mathrm{d} \Gamma / \mathrm{d} K_{e}\right)$ over an energy window $\Delta K_{e}$ scales as $\Delta\left(\mathrm{d} \Gamma / \mathrm{d} K_{e}\right) \propto m_{\beta} / \Delta K_{e}$ [32], which will be vanishing when $\Delta K_{e} \gg m_{\beta}$. If the energy resolution $\Delta=100 \mathrm{meV}$ is larger than absolute neutrino masses, we can evaluate the difference between the effective spectra in the region of $K_{e}<K_{\text {end, } 0}-\Delta$ via series expansion in terms of $m_{i}^{2} / \Delta^{2}$, namely,

$$
\frac{\mathrm{d} \Gamma_{\mathrm{eff}}}{\mathrm{d} K_{e}}-\frac{\mathrm{d} \Gamma_{\mathrm{cl}}^{\prime}}{\mathrm{d} K_{e}} \propto \begin{cases}0, & \text { for } m_{\beta}, \\ m_{\beta}^{\prime 2}-m_{\beta}^{2}, & \text { for } m_{\beta}^{\prime}, \\ m_{\beta}^{\prime \prime 2}-m_{\beta}^{2}, & \text { for } m_{\beta}^{\prime \prime},\end{cases}
$$

where all higher-order terms of $\mathcal{O}\left(m_{i}^{4} / \Delta^{4}\right)$ have been omitted. Consequently, the effective spectrum with $m_{\beta}$ can fit perfectly the experimental observation, whereas a sizable overall shift is left for $m_{\beta}^{\prime}$ as well as for $m_{\beta}^{\prime \prime}$. As we have mentioned before, although the energy resolution is not good enough to completely pin down the end point, the experimental sensitivity to absolute neutrino masses can be obtained by observing the total number of betadecay events within the energy window around the end point. 
An immediate question is whether the effective neutrino mass is still a useful parameter for future beta-decay experiments. Put alternatively, does $\mathrm{d} \Gamma_{\mathrm{cl}}^{\prime} / \mathrm{d} K_{e}$ in Eq. (9) provide a good description of the effective spectrum $\mathrm{d} \Gamma_{\text {eff }} / \mathrm{d} K_{e}$ in Eq. (12)? We will now investigate the validity of the effective neutrino mass by following a simple statistical approach. The strategy of our numerical analysis is summarized as follows.

Given the target mass and the operation time $T$ (i.e., the total exposure $\mathcal{E}$ ), we simulate the experimental data by using the exact spectrum $\mathrm{d} \Gamma_{\mathrm{cl}}^{\prime} / \mathrm{d} K_{e}$ and divide the simulated data into a number of energy bins with bin width $\Delta$, which is taken to be the energy resolution of the detector. In general, the event number in the $i$ th energy bin $\left[E_{i}-\Delta / 2, E_{i}+\Delta / 2\right]$ is given by the integration of the spectrum over the bin width

$$
N_{i}=T \int_{E_{i}-\Delta / 2}^{E_{i}+\Delta / 2} \frac{\mathrm{d} \Gamma_{\Delta}}{\mathrm{d} K_{e}} \mathrm{~d} K_{e}
$$

where $E_{i}$ denotes the mean value of the electron kinetic energy in the $i$ th bin and $\mathrm{d} \Gamma_{\Delta} / \mathrm{d} K_{e}$ is the convolution of a spectrum with a Gaussian smearing function as in Eq. (16). The simulated event number $N_{i}^{\mathrm{cl}}$ in each energy bin is calculated by using $\mathrm{d} \Gamma_{\mathrm{cl}}^{\prime} / \mathrm{d} K_{e}$ with a specified value of $m_{1}$ (i.e., the lightest neutrino mass in the NO case). On the other hand, to clarify how good $\mathrm{d} \Gamma_{\text {eff }} / \mathrm{d} K_{e}$ can describe the true data, the predicted event number $N_{i}^{\text {eff }}$ in each energy bin is calculated in the same way but with the effective spectrum $\mathrm{d} \Gamma_{\text {eff }} / \mathrm{d} K_{e}$, which will be subsequently sent to fit the simulated true data $N_{i}^{\mathrm{cl}}$.

It should be noted that KATRIN operating in the ordinary mode with the MAC-E-Filter observes actually the integrated number of beta-decay events and has to reconstruct the differential spectrum by adjusting the retarding potential to scan over a certain energy window containing the end point. The number of events for the differential spectrum in each energy bin turns out to be $N_{i}=N_{i}^{\text {int }}-N_{i-1}^{\text {int }}$, where $N_{i}^{\text {int }}$ is the event number of the integrated spectrum for the scanning point corresponding to $E_{i}$. For this reason, the statistical fluctuation of the event number for the reconstructed differential spectrum can be estimated as $\sqrt{N_{i}^{\text {int }}+N_{i-1}^{\text {int }}}$, which should be compared with that of $\sqrt{N_{i}}$ for the direct measurement. Meanwhile, a longer time of data taking is also expected. Therefore, our result should be taken to be conservative when considering KATRIN-like experiments operating in integrated mode.

KATRIN can also directly measure the nonintegrated beta spectrum in a possible MAC-E-TOF mode, as described in Appendix A. Since tritium experiments in the future tend to adopt nonintegrated modes to maximize the neutrino mass sensitivity, we shall focus on this scenario. For those tritium experiments operating in the nonintegrated mode, we will use the following experimental configurations: (i) KATRIN with the target mass $m_{\text {KATRIN }}=2.5 \times 10^{-4} \mathrm{~g}$ and energy resolution $\Delta_{\text {KATRIN }}=$ $1 \mathrm{eV}$; (ii) Project 8 loaded with molecular tritium gas with $m_{\mathrm{P} 8}=5 \times 10^{-4} \mathrm{~g}$ and $\Delta_{\mathrm{P} 8 \mathrm{~m}}=0.36 \mathrm{eV}$; (iii) Project 8 loaded with atomic tritium gas with $m_{\mathrm{P} 8}=5 \times 10^{-4} \mathrm{~g}$ and $\Delta_{\mathrm{P} 8 \mathrm{a}}=0.05 \mathrm{eV}$; (iv) PTOLEMY with $m_{\mathrm{PTOLEMY}}=$ $100 \mathrm{~g}$ and $\Delta_{\text {PTOLEMY }}=0.15 \mathrm{eV}$. The details can be found in Appendix A. For Project 8 loaded with molecular tritium, the energy resolution is limited by the irreducible width of the final-state molecular excitations [35]. This limitation can be overcome by switching the target to atomic tritium. Note that for KATRIN in the MAC-E-TOF mode, which is still under development, the penalties of the tritium decay rate and energy resolution due to the chopping procedure (see Appendix A) are ignored, so the configuration here is somewhat idealized for KATRIN. Nevertheless, we will find the effect of using $m_{\beta}$ even in this ideal KATRIN setup is negligible. We adopt Gaussian distributions as in Eq. (16) for the uncertainties caused by finite energy resolutions in all experiments. In a more realistic analysis with all experimental details taken into account, one should consider a strict shape for the energy resolution function, e.g., a trianglelike shape for KATRIN in the developing MAC-E-TOF mode. The actual shape for Project 8 with molecular tritium should also be calculated with detailed consideration of final-state excitations. However, a different shape of the energy resolution from Gaussian should not affect our results by orders of magnitude.

In Fig. 3, we show the difference in the event numbers of $\mathrm{d} \Gamma_{\text {eff }} / \mathrm{d} K_{e}$ and $\mathrm{d} \Gamma_{\mathrm{cl}}^{\prime} / \mathrm{d} K_{e}$, together with the statistical fluctuation of the events. In the upper two panels, two nominal experimental setups have been chosen for demonstration, and in the remaining four panels, we illustrate the cases of realistic experiments. In all panels, the data are simulated with $\mathrm{d} \Gamma_{\mathrm{cl}}^{\prime} / \mathrm{d} K_{e}$, for which a true value of the lightest neutrino mass $m_{1}^{\text {true }}=10 \mathrm{meV}$ has been input, and the data fluctuations are represented by the filled gray histograms. For comparison, the event number difference in each energy bin has been calculated for three effective spectra with $m_{\beta}=13.4 \mathrm{meV}, \quad m_{\beta}^{\prime}=11.9 \mathrm{meV}$, and $m_{\beta}^{\prime \prime}=10 \mathrm{meV}$, which is denoted as the blue dashed curves. In addition, the gray solid curve denotes the exact spectrum with $m_{1}^{\text {true }}=10 \mathrm{meV}$ as in Fig. 2, while the red dotted curve is for $m_{1}^{\text {true }}=10.5 \mathrm{meV}$. From Fig. 3, two important observations can be made. First, for a smaller exposure such as in KATRIN and Project 8, the statistical fluctuation can easily overwhelm the deviations, rendering the effective description of the beta spectrum more reliable. Second, for $m_{\beta}$, the error caused by using the effective spectrum is most significant in the energy bin containing the end point. The reason is obvious, namely that the data fluctuation increases and the deviation decreases, as the energy moves 

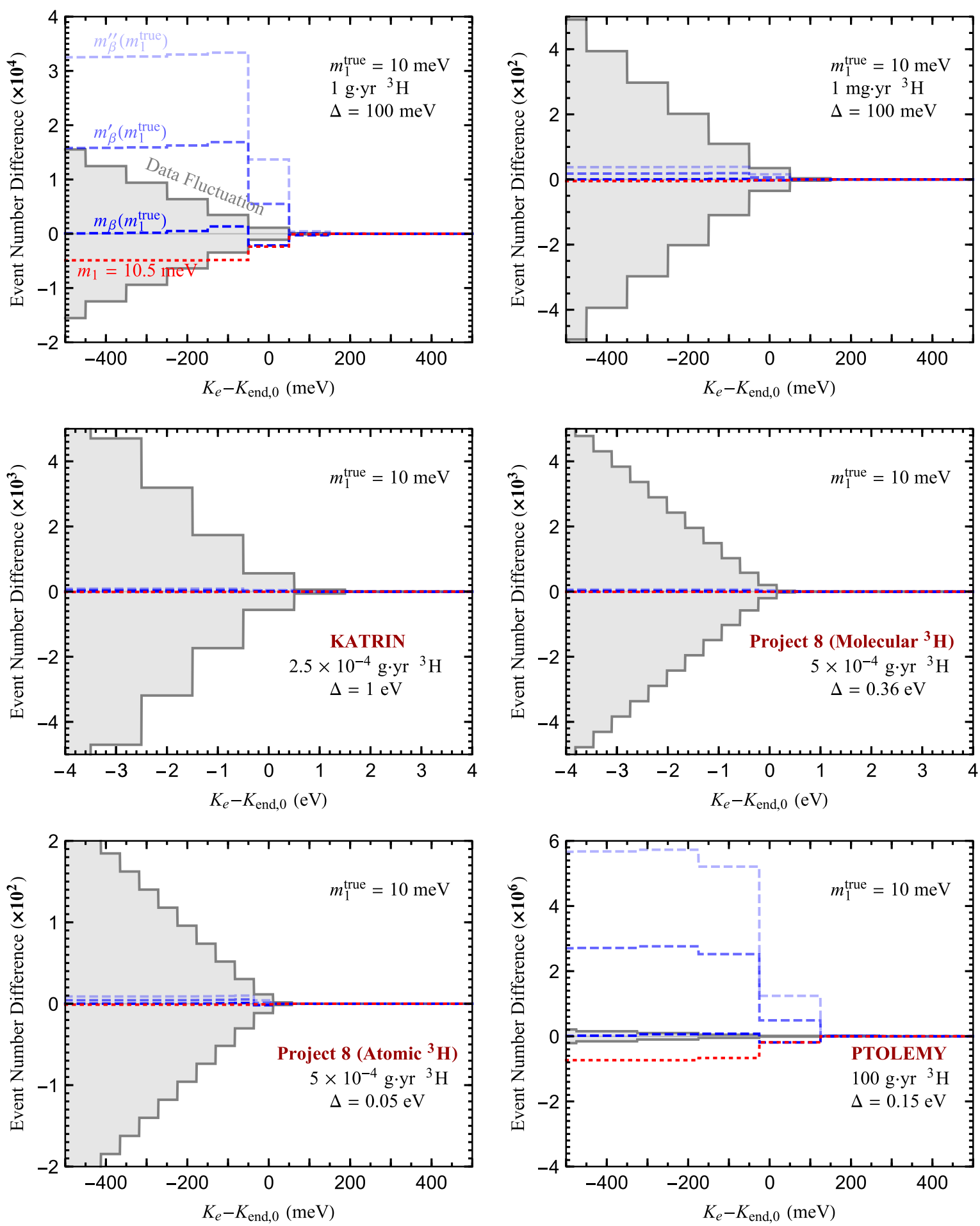

FIG. 3. The difference of event numbers for the effective spectrum $\mathrm{d} \Gamma_{\mathrm{eff}} / \mathrm{d} K_{e}$ and the exact one $\mathrm{d} \Gamma_{\mathrm{cl}}^{\prime} / \mathrm{d} K_{e}$. The data are simulated by taking $m_{1}^{\text {true }}=10 \mathrm{meV}$. The blue histograms signify the event number deviations of the effective spectra from the exact one, whereas the gray filled histograms stand for the statistical fluctuations. Two nominal experimental setups have been assumed in the upper two panels, and in the remaining four panels, we illustrate the cases of realistic experiments including KATRIN, Project 8 with molecular tritium and with atomic one, and PTOLEMY. Note that different scales on the axes have been adopted for each plot. 

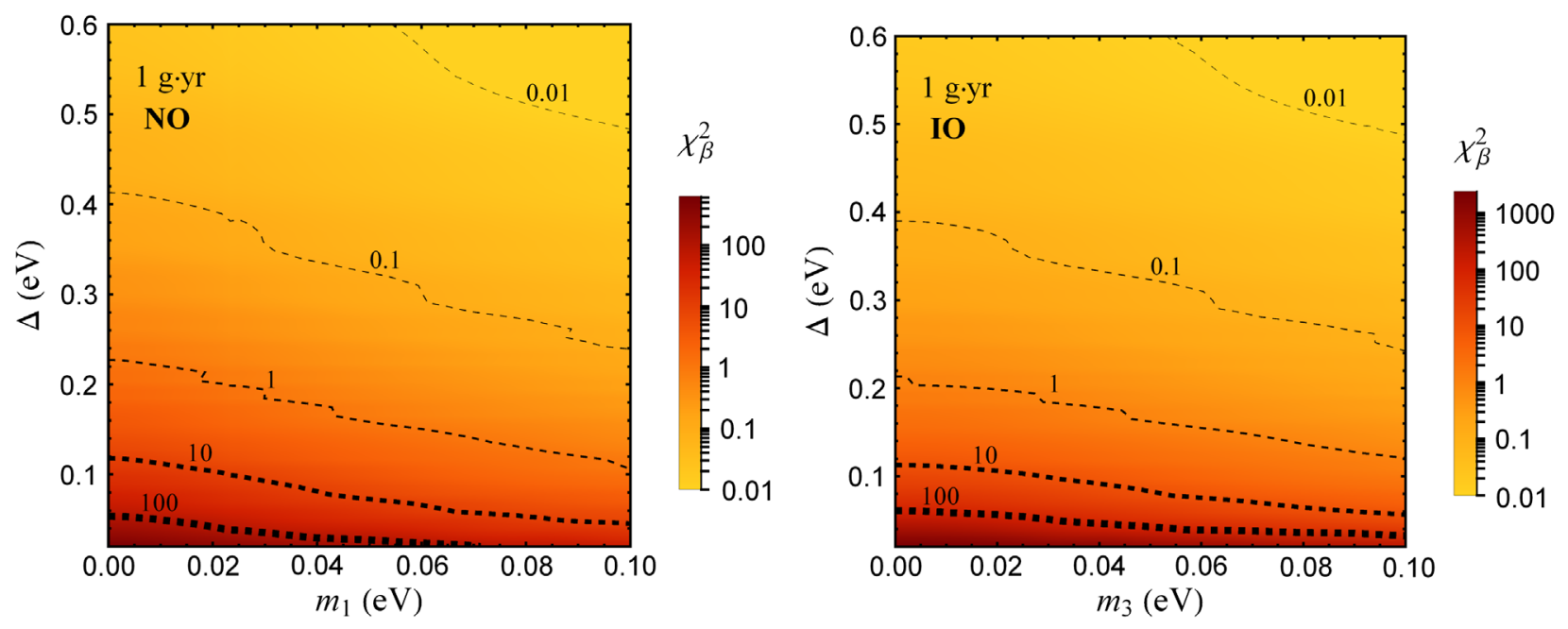

FIG. 4. The contours of $\chi_{\beta}^{2}$, see Eq. (19), arising from the description of the electron spectrum by using the effective neutrino mass $m_{\beta}$, are displayed in the plane of smallest mass versus resolution, i.e., the $m_{1}-\Delta$ plane for the NO case (left panel) and the $m_{3}-\Delta$ plane for the IO case (right panel).

away from the end point. For the other two effective masses $m_{\beta}^{\prime}$ and $m_{\beta}^{\prime \prime}$, the deviations are even more significant.

To quantify the difference between the effective and exact spectra in a statistical approach, we define $\Delta N_{i} \equiv$ $N_{i}^{\text {eff }}-N_{i}^{\text {cl }}$ in each energy bin and take $\sqrt{N_{i}^{\text {cl }}}$ to be the corresponding statistical uncertainty. ${ }^{6}$ In this way, if $\Delta N_{i}$ is negligible compared to $\sqrt{N_{i}^{\mathrm{cl}}}$, one can claim that the error due to the use of the effective spectrum is unimportant in that energy bin. For the whole energy spectrum, the $\chi^{2}$ function can be constructed as

$$
\chi_{\beta}^{2}=\sum_{i} \frac{\left(\Delta N_{i}\right)^{2}}{N_{i}^{\mathrm{cl}}},
$$

where $i$ runs over the number of energy bins. This $\chi^{2}$ function measures to what degree the effective spectrum $\mathrm{d} \Gamma_{\text {eff }} / \mathrm{d} K_{e}$ deviates from the exact one $\mathrm{d} \Gamma_{\mathrm{cl}}^{\prime} / \mathrm{d} K_{e}$. Because we have used $\mathrm{d} \Gamma_{\mathrm{cl}}^{\prime} / \mathrm{d} K_{e}$ to generate the true data, from the model selection perspective (i.e., fitting two different models $\mathrm{d} \Gamma_{\mathrm{cl}}^{\prime} / \mathrm{d} K_{e}$ and $\mathrm{d} \Gamma_{\text {eff }} / \mathrm{d} K_{e}$ with the same data, respectively), $\chi_{\beta}^{2}$ defines the statistical significance with which one can favor $\mathrm{d} \Gamma_{\mathrm{cl}}^{\prime} / \mathrm{d} K_{e}$ over $\mathrm{d} \Gamma_{\text {eff }} / \mathrm{d} K_{e}$. If one insists in using the effective spectrum $\mathrm{d} \Gamma_{\text {eff }} / \mathrm{d} K_{e}$ to fit the data, $\chi_{\beta}^{2}$ also measures the goodness-of-fit $\chi_{\beta}^{2} / v$ of $\mathrm{d} \Gamma_{\text {eff }} / \mathrm{d} K_{e}$ given the degree of freedom $v$ in fitting. Since most deviations of $\mathrm{d} \Gamma_{\text {eff }} / \mathrm{d} K_{e}$ from $\mathrm{d} \Gamma_{\mathrm{cl}}^{\prime} / \mathrm{d} K_{e}$ distribute only in a few energy bins around the end point, the degree of freedom can be $v=\mathcal{O}(1)$ depending on the number of bins we use in the actual fit. As has been mentioned

\footnotetext{
${ }^{6}$ This is true when the event number in each energy bin is large, such that the fluctuation follows approximately a Gaussian distribution, which turns out to be true for all tritium experiments in our consideration as can be noticed in Fig. 3.
}

previously, we have fixed the bin size to be the energy resolution $\Delta$. In principle, the bin width can be chosen freely. The smaller the bin width is, the more information one can acquire in the fit. However, this is limited by the energy resolution of an experiment, which will smooth out the information within a comparable bin size, such that further decreasing the bin size will not improve the result anymore. We have numerically checked that by choosing a bin width smaller than the energy resolution, e.g., $\Delta / 8$, the $\chi^{2}$-function defined in Eq. (19) will increase only by a factor of $\sim 70 \%$. Further reducing the bin width will not alter this result.

Let us make some remarks on the other input in our numerical calculations. First, the best-fit values of neutrino oscillation parameters from Ref. [34] are adopted. Second, the energy window for the analysis has been taken to be $K_{e}-K_{\text {end, } 0} \in(-4 \ldots 4) \mathrm{eV}$. Third, we have assumed no background contributions. The inclusion of possible background events will reduce the value of $\chi_{\beta}^{2}$, leading to a smaller statistical deviation of the effective spectrum $\mathrm{d} \Gamma_{\text {eff }} / \mathrm{d} K_{e}$ from the classical one $\mathrm{d} \Gamma_{\mathrm{cl}}^{\prime} / \mathrm{d} K_{e}$. Four, we take the normalization factor to be one, as it can be precisely determined by choosing a wider energy window in realistic experiments.

In the left panel of Fig. 4, for each pair of $m_{1}$ in the range of $(0 \ldots 0.1) \mathrm{eV}$ and $\Delta$ in the range of $(0.02 \ldots 0.6) \mathrm{eV}$, we present the value of $\chi_{\beta}^{2}$ for the total exposure of $\mathcal{E}=1 \mathrm{~g} \cdot \mathrm{yr}$ in the NO case, where the effective spectrum with $m_{\beta}$ is adopted for illustration. Similar calculations have also been carried out in the IO case and the results are given in the $m_{3}-\Delta$ plane in the right panel. Roughly speaking, for those values of $\Delta$ and $m_{1}$ in the NO case (or $m_{3}$ in the IO case) corresponding to $\chi_{\beta}^{2} \lesssim 0.1$, the effective spectrum $\mathrm{d} \Gamma_{\text {eff }} / \mathrm{d} K_{e}$ with $m_{\beta}$ is reasonably good to describe the data, 
i.e., with negligible and fragile statistical significance to discriminate $\mathrm{d} \Gamma_{\mathrm{cl}}^{\prime} / \mathrm{d} K_{e}$ from $\mathrm{d} \Gamma_{\text {eff }} / \mathrm{d} K_{e}$ and no noticeable impact on the goodness of fit. In the same sense, we can also conclude that $m_{\beta}$ is no longer a safe parameter for those values of $\Delta$ and $m_{1}$ (or $m_{3}$ ) corresponding to $\chi_{\beta}^{2} \gtrsim 10$, i.e., the statistical power to favor $\mathrm{d} \Gamma_{\mathrm{cl}}^{\prime} / \mathrm{d} K_{e}$ over $\mathrm{d} \Gamma_{\text {eff }} / \mathrm{d} K_{e}$ is more than $3 \sigma$ and a considerable impact on the goodnessof-fit arises (the $p$ value of fit is 0.001565 for $\chi_{\beta}^{2}=10$ and $v=1$, and the model $\mathrm{d} \Gamma_{\text {eff }} / \mathrm{d} K_{e}$ is almost ruled out by the data).

Although we have fixed the exposure at $\mathcal{E}=1 \mathrm{~g} \cdot \mathrm{yr}$, it is straightforward to derive the values of $\chi_{\beta}^{2}$ for a different exposure by noting the fact that $\left(\Delta N_{i}\right)^{2} / N_{i}^{\text {cl }}$ is linearly proportional to $\mathcal{E}$. As a consequence, for a different exposure $\tilde{\mathcal{E}}$, the original values of $\chi_{\beta}^{2}$ for $\mathcal{E}$ will be modified to be $\chi_{\beta}^{2} \cdot \tilde{\mathcal{E}} / \mathcal{E}$. For instance, the original value of the contour $\chi_{\beta}^{2}=10$ for $\mathcal{E}=1 \times \mathrm{g} \cdot \mathrm{yr}$ should be changed to $\chi_{\beta}^{2}=0.01$ for $\mathcal{E}=1 \mathrm{mg} \cdot \mathrm{yr}$. Nevertheless, if we insist on using $\mathrm{d} \Gamma_{\text {eff }} / \mathrm{d} K_{e}$ to fit the data regardless of the statistical preference for the true model $\mathrm{d} \Gamma_{\mathrm{cl}}^{\prime} / \mathrm{d} K_{e}$ and a poor goodness of fit, the parameter estimation of $m_{\beta}$ can always be performed based on using $\mathrm{d} \Gamma_{\text {eff }} / \mathrm{d} K_{e}$. In this case, a large value of $\chi_{\beta}^{2}$ does not necessarily mean a large value of $\Delta \chi^{2} \equiv \chi_{\beta}^{2}-\left.\chi_{\beta}^{2}\right|_{\text {min }}$ in parameter estimations, where $\left.\chi_{\beta}^{2}\right|_{\text {min }}$ denotes the minimum of $\chi_{\beta}^{2}$ by freely adjusting $m_{\beta}$ or $m_{1}$.

To explicitly show the error of fitting, the neutrino mass $m_{1}$ with the effective spectrum, we calculate $\Delta \chi^{2}$ and present the final result with respect to $m_{1}$ in Fig. 5. In our calculations, we assume the true value of $m_{1}$ to be $10 \mathrm{meV}$, corresponding to $m_{\beta}=13.4 \mathrm{meV}$. The energy resolution is fixed to $0.1 \mathrm{eV}$. The dark red curve represents the result obtained by fitting with the exact spectrum $\mathrm{d} \Gamma_{\mathrm{cl}}^{\prime} / \mathrm{d} K_{e}$, while the dark blue one corresponds to the fit by using the effective spectrum $\mathrm{d} \Gamma_{\text {eff }} / \mathrm{d} K_{e}$, given the exposure of $\mathcal{E}=100 \mathrm{~g} \cdot \mathrm{yr}$. The light curves stand for the case with $\mathcal{E}=1 \mathrm{~g} \cdot \mathrm{yr}$. One can observe that if the effective spectrum with $m_{\beta}$ is used, the best-fit value of $m_{1}$ is found to be $m_{1}^{\text {bf }}=9.6 \mathrm{meV}$, which deviates notably from $m_{1}^{\text {bf }}=10 \mathrm{meV}$ obtained by using the exact spectrum. Even for the exposure of $\mathcal{E}=1 \mathrm{~g} \cdot \mathrm{yr}$, the true value is outside of $\Delta \chi^{2} \lesssim 4$ when fitting with the effective spectrum. The situation becomes worse if we take a larger exposure.

To systematically study how far the parameter value fitted by using $\mathrm{d} \Gamma_{\text {eff }} / \mathrm{d} K_{e}$ can deviate from the true one, we define the following difference of $\chi^{2}$ :

$$
\Delta \chi_{\text {true }}^{2} \equiv \chi_{\beta}^{2}\left(m_{\beta}=m_{\beta}^{\text {true }}\right)-\left.\chi_{\beta}^{2}\left(m_{\beta}=m_{\beta}^{\text {bf }}\right)\right|_{\min },
$$

where $\chi_{\beta}^{2}\left(m_{\beta}=m_{\beta}^{\text {true }}\right)$ is the $\chi^{2}$ value when $m_{\beta}$ is set to $m_{\beta}^{\text {true }}$ when fitting with $\mathrm{d} \Gamma_{\text {eff }} / \mathrm{d} K_{e}$, and $\left.\chi_{\beta}^{2}\left(m_{\beta}=m_{\beta}^{\mathrm{bf}}\right)\right|_{\min }$ is the minimum value of the $\chi^{2}$ curve obtained by freely adjusting

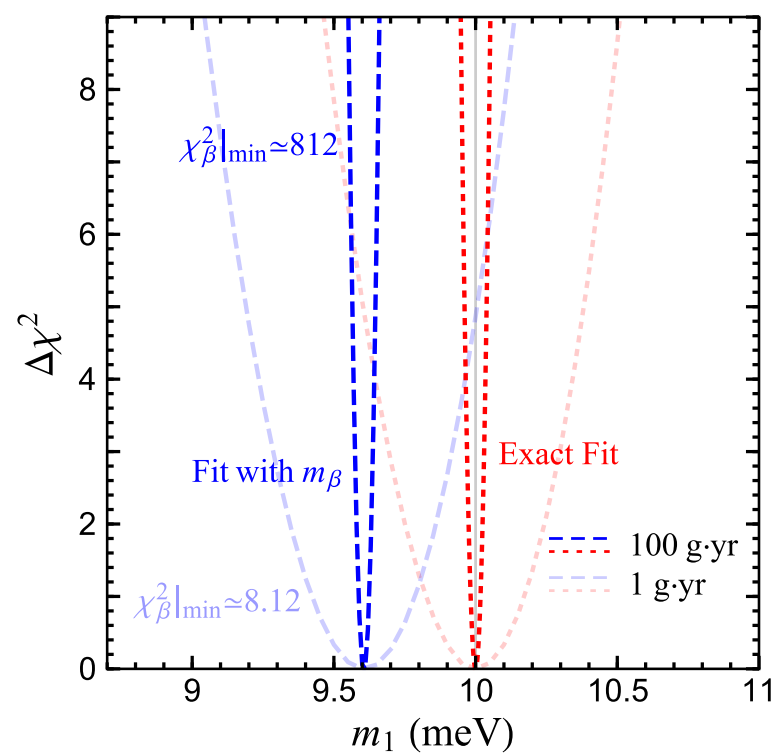

FIG. 5. The function $\Delta \chi^{2} \equiv \chi_{\beta}^{2}-\left.\chi_{\beta}^{2}\right|_{\min }$ is shown with respect to the lightest neutrino mass $m_{1}$ in the NO case. The dark red curve is generated by fitting with the exact spectrum $\mathrm{d} \Gamma_{\mathrm{cl}}^{\prime} / \mathrm{d} K_{e}$, while the dark blue one is by using the effective spectrum $\mathrm{d} \Gamma_{\text {eff }} / \mathrm{d} K_{e}$ with $m_{\beta}$, for the exposure of $\mathcal{E}=100 \mathrm{~g} \cdot \mathrm{yr}$. The light curves are for $\mathcal{E}=1 \mathrm{~g} \cdot \mathrm{yr}$ with all else being the same. For illustration, we use an energy resolution of $\Delta=0.1 \mathrm{eV}$.

$m_{\beta}$ with $m_{\beta}^{\text {bf }}$ being the best-fit value. The value of $m_{\beta}^{\text {true }}$ can be directly obtained with Eq. (1) once the input value of $m_{1}$ in $\mathrm{d} \Gamma_{\mathrm{cl}}^{\prime} / \mathrm{d} K_{e}$ for simulating the data is given. The difference $\Delta \chi_{\text {true }}^{2}$ measures how likely one can recover the true value of the model parameter $m_{\beta}$ by fitting with $\mathrm{d} \Gamma_{\text {eff }} / \mathrm{d} K_{e}$. We present $\chi_{\beta}^{2}$ and $\Delta \chi_{\text {true }}^{2}$ in Fig. 6 as a function of the exposure $\mathcal{E}$ and the energy resolution $\Delta$. We fix the lightest neutrino mass as $0 \mathrm{eV}$ for these plots, as $\chi_{\beta}^{2}$ is maximized in this case according to Fig. 4.

The experimental configurations of KATRIN, Project 8, and PTOLEMY have been indicated in Fig. 6, and their corresponding $\chi^{2}$ values have been explicitly summarized in Table I. For PTOLEMY, the effective beta spectrum can no longer be adopted. The use of the effective spectrum with $m_{\beta}$ would result in a huge error in fitting the neutrino mass compared to the precision that is supposed to be achieved in such an experiment, e.g., $\Delta \chi_{\text {true }}^{2}=141$ for $\mathrm{NO}$ and $\Delta \chi_{\text {true }}^{2}=81$ for IO for 1 year of data taking. For KATRIN and Project 8, with 1 year of exposure, the effective mass $m_{\beta}$ is fortunately applicable with $\chi_{\beta}^{2}$, $\Delta \chi_{\text {true }}^{2} \lesssim 0.1$. Note that there is a little risk for Project 8 loaded with the atomic tritium. To be more specific, in the extreme case that the data taking time is set to 10 years and an improvement on the energy resolution is made to $\Delta=0.03 \mathrm{eV}, \Delta \chi_{\text {true }}^{2}$ for NO can be as large as 1, indicating that the true value of $m_{\beta}$ is out of the $1 \sigma \mathrm{CL}$ region by fitting with the effective spectrum $\mathrm{d} \Gamma_{\text {eff }} / \mathrm{d} K_{e}$; hence, the 

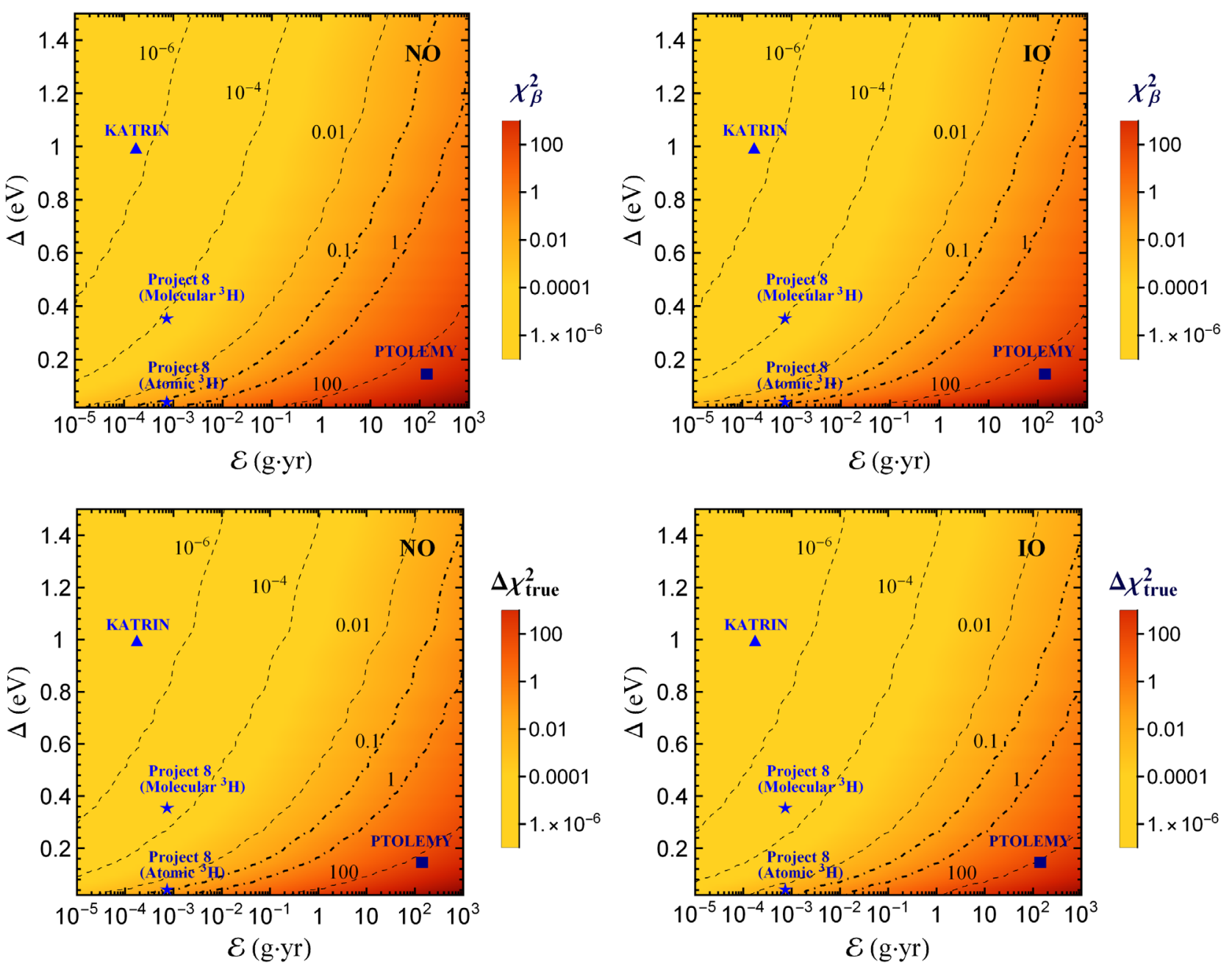

FIG. 6. The contours of $\chi_{\beta}^{2}$ and $\Delta \chi_{\text {true }}^{2}$, see Eqs. (19) and (20), arising from the description of the electron spectrum by using the effective neutrino mass $m_{\beta}$, are displayed in the exposure-resolution $(\mathcal{E}-\Delta)$ plane for the NO case (left two panels) and the IO case (right two panels). The lightest neutrino mass is fixed to $0 \mathrm{eV}$.

description by using the effective spectrum would not be appropriate anymore.

\section{POSTERIOR DISTRIBUTIONS}

As we have already demonstrated in the previous section, the effective spectrum $\mathrm{d} \Gamma_{\text {eff }} / \mathrm{d} K_{e}$ cannot be used for PTOLEMY, but is safe to use in the KATRIN and Project 8 experiments. Following the Bayesian statistical approach [36], we derive in this section the posterior distributions of the effective neutrino mass $m_{\beta}$, based on current experimental information from neutrino oscillations, beta decay, neutrinoless double-beta decay $(0 \nu \beta \beta)$, and cosmology. Since the description of the beta spectrum via the effective neutrino mass is still valid for KATRIN and Project 8, posterior distributions of the effective neutrino mass should be very suggestive for future experiments. Our results in this section can also be used for the electron-capture experiments ECHo [14], HOLMES [15], and NuMECS [16], if $C P T$ is assumed to be conserved in the neutrino sector. For the similar analysis relevant for the effective neutrino masses in $\beta$ and $0 \nu \beta \beta$ decays, see Refs. [37-43]. Here we perform an updated analysis for the direct neutrino mass experiments, in light of a good number of experimental achievements.

As usual, two important ingredients for the Bayesian analysis should be specified. First, we have to choose the prior distributions for the relevant model parameters

$$
\left\{\sin ^{2} \theta_{12}, \sin ^{2} \theta_{13}, \Delta m_{\mathrm{sol}}^{2}, \Delta m_{\mathrm{atm}}^{2}, \rho, \sigma, G_{0 \nu},\left|\mathcal{M}_{0 \nu}\right|, m_{\mathrm{L}}\right\},
$$

where $\Delta m_{\mathrm{sol}}^{2}=\Delta m_{21}^{2}$ and $\Delta m_{\mathrm{atm}}^{2}=\Delta m_{31}^{2}$ (or $\Delta m_{32}^{2}$ ) in the NO (or IO) case. For all oscillation parameters $\left\{\sin ^{2} \theta_{12}, \sin ^{2} \theta_{13}, \Delta m_{\text {sol }}^{2}, \Delta m_{\text {atm }}^{2}\right\}$, we assume that they are uniformly distributed in the ranges that are wide enough to cover their experimentally allowed values. For the absolute neutrino mass scale, which is represented by the lightest neutrino mass $m_{\mathrm{L}}$ (i.e., $m_{1}$ in the NO case or 

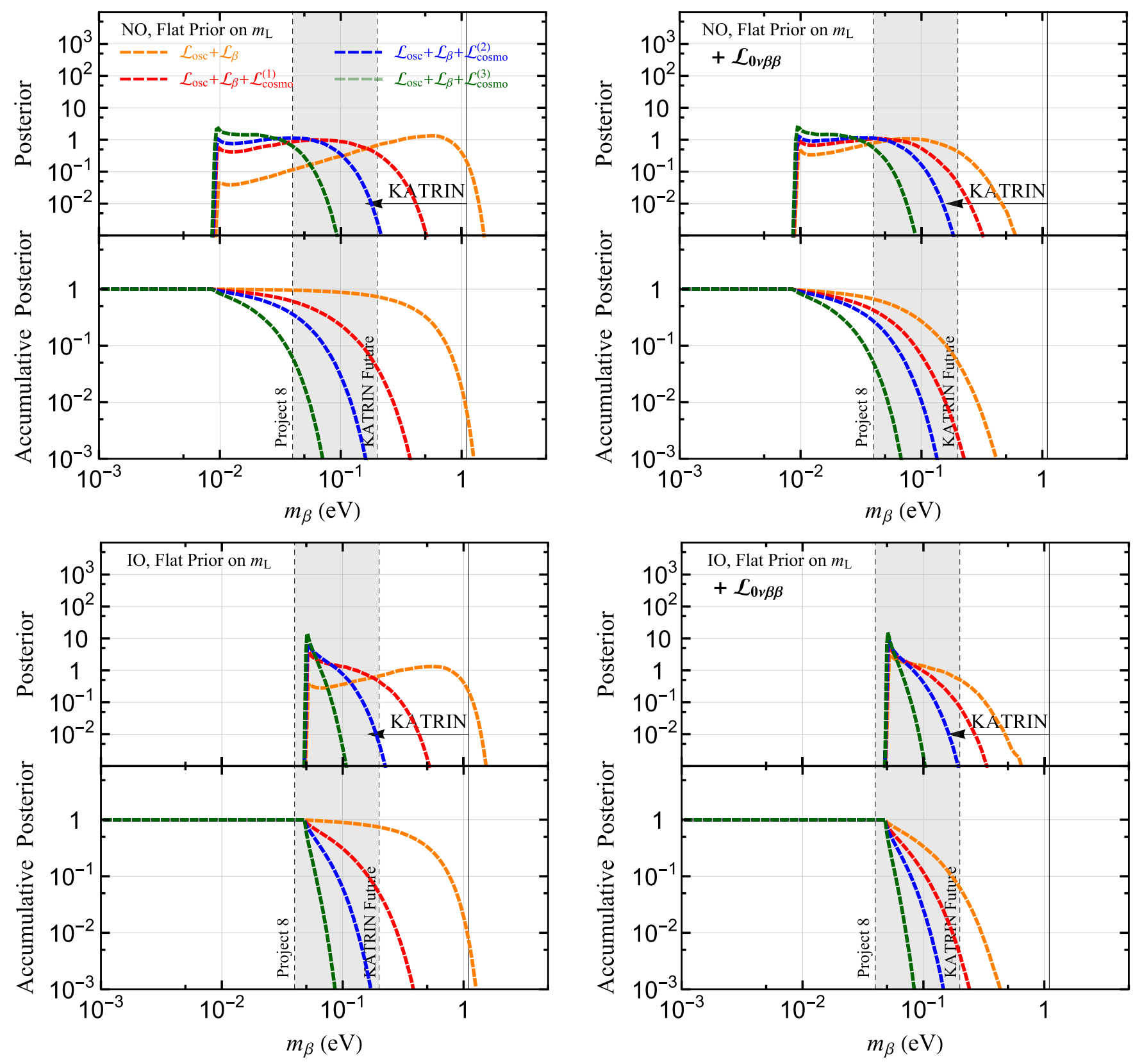

FIG. 7. The posterior distributions of the effective neutrino mass $m_{\beta}$ in the NO (upper row) and IO (lower row) cases, given a flat prior on the lightest neutrino mass $m_{\mathrm{L}}$. In each of the four subfigures, the upper subgraph shows the posterior distributions whereas the lower subgraph gives the accumulative distributions. The results for four different combinations of experimental information have been displayed in the left column: (i) $\mathcal{L}_{\text {osc }}+\mathcal{L}_{\beta}$ (orange curves); (ii) $\mathcal{L}_{\text {osc }}+\mathcal{L}_{\beta}+\mathcal{L}_{\text {cosmo }}^{(1)}$ (red curves); (iii) $\mathcal{L}_{\text {osc }}+\mathcal{L}_{\beta}+\mathcal{L}_{\text {cosmo }}^{(2)}$ (blue curves); (iv) $\mathcal{L}_{\text {osc }}+\mathcal{L}_{\beta}+\mathcal{L}_{\text {cosmo }}^{(3)}$ (green curves), while the data from $0 \nu \beta \beta$ using $\mathcal{L}_{0 \nu \beta \beta}$ are further included in the right column. The cosmological bounds on the sum of three neutrino masses corresponding to $\mathcal{L}_{\text {cosmo }}^{(i)}$ (for $i=1,2,3$ ) have been summarized in Eq. (B5). The latest result $m_{\beta}<1.1 \mathrm{eV}$ from KATRIN is denoted as the vertical solid line, and future sensitivities of KATRIN and Project 8 are represented by two vertical dashed lines.

$m_{3}$ in the IO case), we consider the following two possible priors:

(i) A flat prior on the logarithm of $m_{\mathrm{L}}$ in the range of $\left(10^{-7} \cdots 10\right) \mathrm{eV}$, namely, $\log _{10}\left(m_{\mathrm{L}} / \mathrm{eV}\right) \in[-7,1]$ which will be referred to as the log prior in the following discussion. This prior is scale invariant and motivated by the approximately constant ratios [44] of charged fermion masses $m_{\mathrm{u}} / m_{\mathrm{c}} \sim m_{\mathrm{c}} / m_{\mathrm{t}} \sim$ $\lambda^{2}, m_{\mathrm{d}} / m_{\mathrm{s}} \sim m_{\mathrm{s}} / m_{\mathrm{b}} \sim \lambda$, and $m_{e} / m_{\mu} \sim m_{\mu} / m_{\tau} \sim \lambda^{2}$ (where $\lambda=\sin \theta_{\mathrm{C}} \approx 0.22$ is the Wolfenstein parameter), as well as by the in general exponential fermion mass hierarchies. Note that an ad hoc lower 

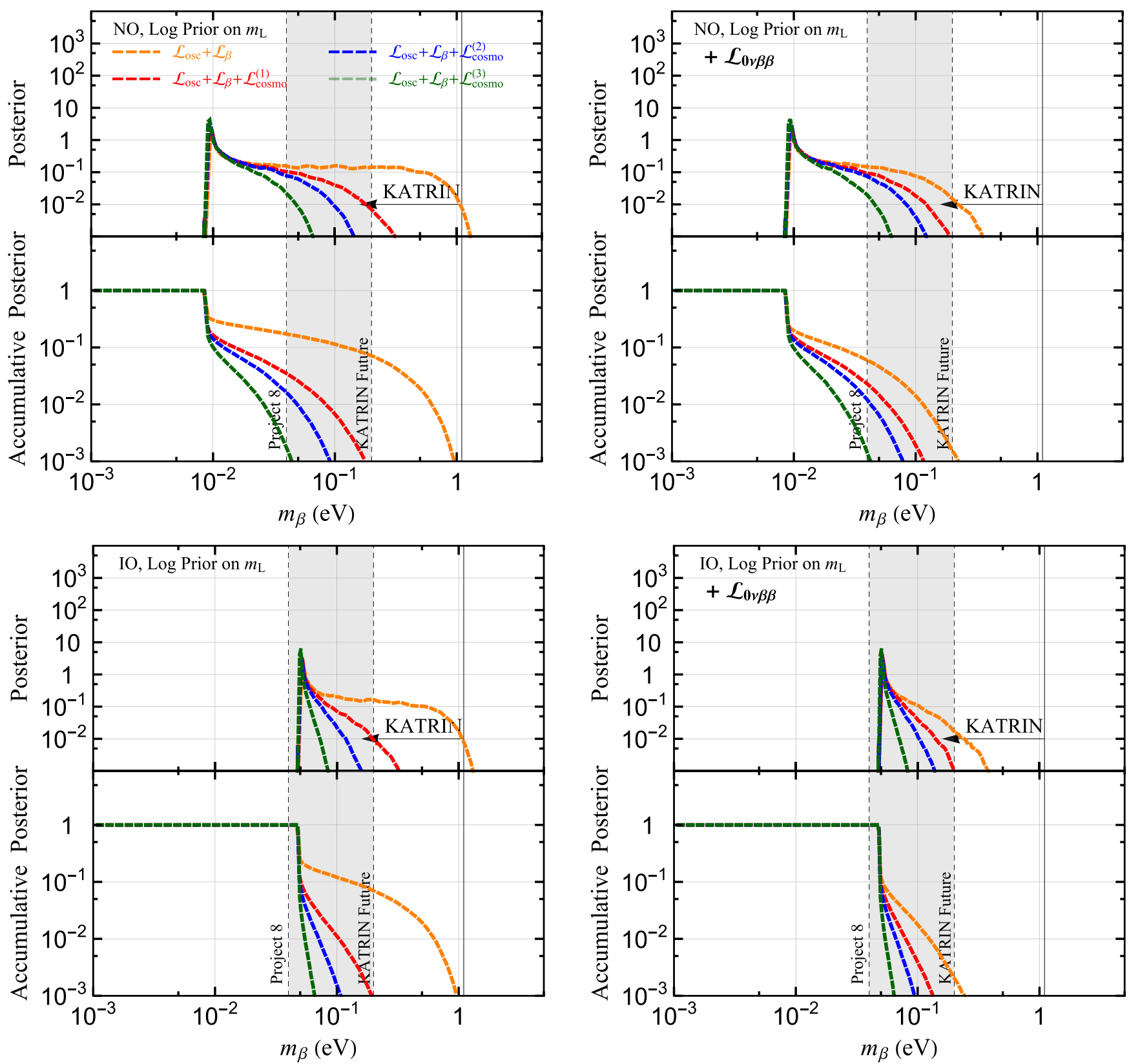

FIG. 8. The posterior distributions of the effective neutrino mass $m_{\beta}$ in the NO (upper row) and IO (lower row) cases, given a log prior on the lightest neutrino mass $m_{\mathrm{L}}$. In each of the four subfigures, the upper subgraph shows the posterior distributions whereas the lower subgraph gives the accumulative distributions. The results for four different combinations of experimental information have been displayed in the left column: (i) $\mathcal{L}_{\text {osc }}+\mathcal{L}_{\beta}$ (orange curves); (ii) $\mathcal{L}_{\text {osc }}+\mathcal{L}_{\beta}+\mathcal{L}_{\text {cosmo }}^{(1)}$ (red curves); (iii) $\mathcal{L}_{\text {osc }}+\mathcal{L}_{\beta}+\mathcal{L}_{\text {cosmo }}^{(2)}$ (blue curves); (iv) $\mathcal{L}_{\text {osc }}+\mathcal{L}_{\beta}+\mathcal{L}_{\text {cosmo }}^{(3)}$ (green curves), while the data from $0 \nu \beta \beta$ using $\mathcal{L}_{0 \nu \beta \beta}$ are further included in the right column. The cosmological bounds on the sum of three neutrino masses corresponding to $\mathcal{L}_{\text {cosmo }}^{(i)}$ (for $i=1,2,3$ ) have been summarized in Eq. (B5). The latest result $m_{\beta}<1.1 \mathrm{eV}$ from KATRIN is denoted as the vertical solid line, and future sensitivities of KATRIN and Project 8 are represented by two vertical dashed lines.

cutoff $10^{-7} \mathrm{eV}$ for $m_{\mathrm{L}}$ has been imposed, which is necessary to bound the prior volume from below. Decreasing this cutoff is equivalent to putting more and more prior volume to very small and essentially vanishing values of $m_{\mathrm{L}}$.

(ii) A flat prior on $m_{\mathrm{L}}$ in the range of $(0 \ldots 10) \mathrm{eV}$. Note that the ratio of the heaviest to the second- heaviest neutrino mass is rather small, at most $\sqrt{\Delta m_{31}^{2} / \Delta m_{21}^{2}} \approx 5$ for $\mathrm{NO}$ and essentially 1 for IO, motivating a moderate and nonexponential ordering of neutrino masses.

Without a complete theory for neutrino mass generation, we cannot judge which prior is favorable and therefore shall treat both of them on equal footing. The prior dependence 
TABLE II. The volume fraction of the $m_{\beta}$ posterior covered by KATRIN with a sensitivity of $m_{\beta} \simeq 0.2 \mathrm{eV}$ and Project 8 with a sensitivity of $m_{\beta} \simeq 0.04 \mathrm{eV}$, respectively. A flat and logarithmic prior on the lightest neutrino mass has been assumed for the upper and lower tables, respectively.

\begin{tabular}{lcccccccc}
\hline \hline Flat prior & $\mathcal{L}_{\mathrm{osc}}+\mathcal{L}_{\beta}$ & $+\mathcal{L}_{\text {cosmo }}^{(1)}$ & $+\mathcal{L}_{\text {cosmo }}^{(2)}$ & $+\mathcal{L}_{\text {cosmo }}^{(3)}$ & $+\mathcal{L}_{0 \nu \beta \beta}$ & $+\mathcal{L}_{0 \nu \beta \beta}+\mathcal{L}_{\text {cosmo }}^{(1)}$ & $+\mathcal{L}_{0 \nu \beta \beta}+\mathcal{L}_{\text {cosmo }}^{(2)}+\mathcal{L}_{0 \nu \beta \beta}+\mathcal{L}_{\text {cosmo }}^{(3)}$ \\
\hline KATRIN, NO & $73 \%$ & $4.2 \%$ & $6.9 \times 10^{-5}$ & $<10^{-11}$ & $4.7 \%$ & $0.23 \%$ & $5.2 \times 10^{-6}$ & $<10^{-11}$ \\
KATRIN, IO & $74 \%$ & $4.9 \%$ & $1.2 \times 10^{-4}$ & $<10^{-11}$ & $6.5 \%$ & $0.45 \%$ & $1.2 \times 10^{-5}$ & $4.9 \%$ \\
Project 8, NO & $96 \%$ & $60 \%$ & $35 \%$ & $6 \%$ & $67 \%$ & $43 \%$ & $10^{-11}$ & $28 \%$ \\
Project 8, IO & $100 \%$ & $100 \%$ & $100 \%$ & $100 \%$ & $100 \%$ & $100 \%$ & $100 \%$ & $100 \%$ \\
Log prior & $\mathcal{L}_{\mathrm{osc}}+\mathcal{L}_{\beta}$ & $+\mathcal{L}_{\text {cosmo }}^{(1)}$ & $+\mathcal{L}_{\text {cosmo }}^{(2)}$ & $+\mathcal{L}_{\text {cosmo }}^{(3)}$ & $+\mathcal{L}_{0 \nu \beta \beta}$ & $+\mathcal{L}_{0 \nu \beta \beta}+\mathcal{L}_{\text {cosmo }}^{(1)}+\mathcal{L}_{0 \nu \beta \beta}+\mathcal{L}_{\text {cosmo }}^{(2)}+\mathcal{L}_{0 \nu \beta \beta}+\mathcal{L}_{\text {cosmo }}^{(3)}$ \\
KATRIN, NO & $7.2 \%$ & $6.8 \times 10^{-4}$ & $7.9 \times 10^{-7}$ & $<10^{-11}$ & $0.15 \%$ & $3.7 \times 10^{-5}$ & $6.4 \times 10^{-8}$ & $<10^{-11}$ \\
KATRIN, IO & $7.1 \%$ & $9.3 \times 10^{-4}$ & $1.4 \times 10^{-6}$ & $<10^{-11}$ & $0.19 \%$ & $6.6 \times 10^{-5}$ & $1.7 \times 10^{-7}$ & $<10^{-11}$ \\
Project 8, NO & $17 \%$ & $3.5 \%$ & $1.6 \%$ & $0.17 \%$ & $6.0 \%$ & $2.3 \%$ & $1.2 \%$ & $10 \%$ \\
Project 8, IO & $100 \%$ & $100 \%$ & $100 \%$ & $100 \%$ & $100 \%$ & $100 \%$ & $10 \%$ \\
\hline \hline
\end{tabular}

of the final posterior distributions reflects that current experimental knowledge on the absolute scale of neutrino masses is still very poor. If one attempts to set limits on model parameters, a prior-independent approach may be found in Ref. [45].

The likelihood functions for each type of experiments can be found in Appendix B. Briefly speaking, the globalfit results of all neutrino oscillation data from Ref. [34] will be used to construct the likelihood function $\mathcal{L}_{\text {osc }}\left(\sin ^{2} \theta_{12}, \sin ^{2} \theta_{13}, \Delta m_{\text {sol }}^{2}, \Delta m_{\text {atm }}^{2}\right)$. The tritium betadecay experiments Mainz [12], Troitsk [13], and KATRIN [9] are taken into account and the likelihood function $\mathcal{L}_{\beta}\left(m_{\beta}^{2}\right)$ involves the model parameters $\left\{m_{\mathrm{L}}\right.$, $\left.\sin ^{2} \theta_{12}, \sin ^{2} \theta_{13}, \Delta m_{\text {sol }}^{2}, \Delta m_{\mathrm{atm}}^{2}\right\}$. As for $0 \nu \beta \beta$ experiments, the likelihood function $\mathcal{L}_{0 \nu \beta \beta}\left(m_{\beta \beta}, G_{0 \nu},\left|\mathcal{M}_{0 \nu}\right|\right)$ actually contains all the parameters in Eq. (21). For the two Majorana $C P$ phases $\rho$ and $\sigma$ relevant for $0 \nu \beta \beta$ experiments, we shall take flat priors in the range of $[0 \ldots 2 \pi)$, as there is currently no experimental constraint on them. For the phase space factor $G_{0 \nu}$, a Gaussian prior is assumed with the central value and $1 \sigma$ error available from Ref. [46]. The nuclear matrix elements $\left|\mathcal{M}_{0 \nu}\right|$ take a flat prior in the range spanned by the predictions from different NME models [39]. Finally, the upper bound on the sum of three neutrino masses $\Sigma=m_{1}+m_{2}+m_{3}$ from cosmological observations will be implemented, and the corresponding likelihood function $\mathcal{L}_{\text {cosmo }}^{(i)}$ depends on $\left\{m_{\mathrm{L}}, \Delta m_{\text {sol }}^{2}, \Delta m_{\text {atm }}^{2}\right\}$, where $i=1,2,3$ refers, respectively, to the Planck data on the cosmic microwave background, its combination with gravitational lensing data, and their further combination with baryon acoustic oscillation data, as explained in Appendix B.

With the priors of model parameters and the likelihood functions from the relevant experiments, we can compute the posterior distribution of $m_{\beta}$, i.e., $\mathrm{d} \mathcal{P} / \mathrm{d} m_{\beta}$, in the standard way of Bayesian analysis. The sampling is done with the help of the MultiNest routine [47-49]. The numerical results for the flat and $\log$ priors on $m_{\mathrm{L}}$ are shown in Figs. 7 and 8, respectively. A summary of the volume fractions of $m_{\beta}$ posteriors covered by future KATRIN and Project 8 sensitivities has been presented in Table II. Some comments on the numerical results are in order.

(1) In Fig. 7, a flat prior on $m_{\mathrm{L}}$ is assumed. The plots in the first row are for the NO case, whereas those in the second row are for the IO case. In each row, the upper subgraph in the left column shows the posterior distributions of the effective mass in four different scenarios of adopted experimental information: (i) $\mathcal{L}_{\mathrm{osc}}+\mathcal{L}_{\beta}$ for the neutrino oscillation and beta-decay data and (ii) $\mathcal{L}_{\text {osc }}+\mathcal{L}_{\beta}+\mathcal{L}_{\text {cosmo }}^{(i)}$ (for $i=1,2,3$ ) for a further inclusion of cosmological upper bounds on the sum of three neutrino masses. The lower subgraph gives the accumulative posterior distributions, which are defined as $\mathcal{P}\left(m_{\beta}>m_{\beta}^{0}\right)=\int_{m_{\beta}^{0}}^{\infty}\left(\mathrm{d} \mathcal{P} / \mathrm{d} m_{\beta}\right) \mathrm{d} m_{\beta}$. In addition, the plots in the right column differ from those in the left column only by including the experimental information on $0 \nu \beta \beta$. Hence, if neutrinos are Dirac particles, the results including $\mathcal{L}_{0 \nu \beta \beta}$ do not apply. The future sensitivities of KATRIN [11] $(0.2 \mathrm{eV})$ and Project 8 [17] (40 meV) are shown as the upper and lower dashed boundaries of the gray bands. This gray region represents the gradual improvement of the sensitivities. In Fig. 8, the same computations have been carried out for the log prior on $m_{\mathrm{L}}$, where all notations follow those of Fig. 7.

(2) By comparing among the different scenarios in both Figs. 7 and 8, we can make the following important observations:

(a) Let us first focus on the impact of $0 \nu \beta \beta$. If the cosmological observations, namely, the upper bounds on neutrino masses, are not considered, then one can make a comparison between the orange curves (corresponding to $\mathcal{L}_{\text {osc }}+\mathcal{L}_{\beta}$ ) in the left column and those (corresponding to 
$\mathcal{L}_{\text {osc }}+\mathcal{L}_{\beta}+\mathcal{L}_{0 \nu \beta \beta}$ ) in the right column. It is evident that the experimental constraints from $0 \nu \beta \beta$ decays lead to a significant shift of the posterior distribution to the region of smaller values of $m_{\beta}$. Even in this case, it is very likely that the future beta-decay experiments can determine the absolute neutrino mass, no matter whether NO or IO is true. For instance, in Fig. 7, where the flat prior on $m_{\mathrm{L}}$ is assumed, Project 8 can cover $67 \%$ of the posteriors in the NO case.

(b) One should investigate what role is played by the cosmological observations. For this purpose, we concentrate on the plots in the right columns of Figs. 7 and 8. When the cosmological observations are considered, one can see that the probability of discovering a nonzero effective neutrino mass in beta-decay experiments drops dramatically. In the worst situation, where the likelihood set $\mathcal{L}_{\text {osc }}+\mathcal{L}_{\beta}+\mathcal{L}_{\text {cosmo }}^{(3)}+\mathcal{L}_{0 \nu \beta \beta}$ is taken in the NO case, even Project 8 can only cover $4.9 \%$ of the posterior. Therefore, the detection of a positive signal in this case would imply a tension between the beta-decay experiments and cosmological observations.

(c) For the log prior on $m_{\mathrm{L}}$ in Fig. 8, it is easy to recognize that the posterior is remarkably reduced in the region of effective neutrino masses covered by the KATRIN and Project 8 experiments. Compared to the flat prior, this can be interpreted as a consequence of the fact that a large fraction of the prior space has been distributed in the neighborhood of a nearly vanishing $m_{\mathrm{L}}$. For the NO case, the effective neutrino mass $m_{\beta}$ takes the minimum value $\sim 8.9 \times 10^{-3} \mathrm{eV}$ as $m_{\mathrm{L}}=m_{1} \rightarrow 0 \mathrm{eV}$. The beta-decay experiments like KATRIN and Project 8 are still far from achieving this sensitivity.

Regardless of the prior and likelihood choices, Project 8 can always cover all the posteriors of the IO case. In this connection, the discrimination between NO and IO seems to be very promising in future beta-decay experiments [50], e.g., an explicit study of the sensitivity has already been performed for PTOLEMY in Ref. [20].

\section{SUMMARY}

The determination of absolute neutrino masses is experimentally challenging, but scientifically very important. As fundamental parameters in nature, absolute neutrino masses must be precisely measured in order to explore the origin of neutrino masses, which calls for new physics beyond the standard model. Motivated by the latest result from the KATRIN experiment and upcoming tritium beta-decay searches, we have performed a detailed study of the exact electron spectrum $\mathrm{d} \Gamma_{\mathrm{cl}}^{\prime} / \mathrm{d} K_{e}$ in Eq. (9), which is a modified relativistic one, and its difference to the effective electron spectrum $\mathrm{d} \Gamma_{\text {eff }} / \mathrm{d} K_{e}$ in Eq. (12) which includes the usually considered effective neutrino mass $m_{\beta}$ or its variants. Moreover, based on current experimental information from neutrino oscillation data, tritium beta decays, neutrinoless double-beta decays, and cosmology, we have computed the posterior distributions of the effective neutrino mass $m_{\beta}$ in Eq. (1). Our main results are summarized as follows.

First, for tritium beta decays, the classical electron spectrum $\mathrm{d} \Gamma_{\mathrm{cl}} / \mathrm{d} K_{e}$ can be modified by replacing $m_{i}$ with $m_{i} \cdot\left(m_{3} \mathrm{He} / m_{3 \mathrm{H}}\right)$ to account for the exact electron spectrum including relativistic corrections. In this case, the difference between the exact relativistic spectrum and the modified classical spectrum $\mathrm{d} \Gamma_{\mathrm{cl}}^{\prime} / \mathrm{d} K_{e}$ can be safely ignored, as the dominant uncertainties in the measurements at KATRIN, Project 8, and PTOLEMY arise from the statistical data fluctuations. Furthermore, it is interesting to compare the exact spectrum with the effective one containing the usually considered observable $m_{\beta}$. However, as we have demonstrated in a quantitative way, the validity of the effective mass $m_{\beta}$ actually depends on the energy resolution and the total exposure of a realistic beta-decay experiment. We show that the use of the standard effective neutrino mass for KATRIN and Project 8 is justified. For the future PTOLEMY experiment with an exposure of $100 \mathrm{~g} \cdot \mathrm{yr}$, it will be problematic to introduce an effective neutrino mass, and the lightest neutrino mass should be used together with the exact spectrum. While this is known, we have performed here a general analysis with keeping the exposure and energy resolution as free parameters.

Second, as we have mentioned above, it is justified to describe the exact electron spectrum $\mathrm{d} \Gamma_{\mathrm{cl}}^{\prime} / \mathrm{d} K_{e}$ by the effective one with the effective neutrino mass $m_{\beta}$ in the KATRIN and Project 8 experiments. Therefore, it does make sense to derive the posterior distributions of the effective neutrino mass, given the latest experimental data on neutrino oscillations, beta decays, neutrinoless double-beta decays, and cosmological observations. Although the cosmological upper bound on the sum of three neutrino masses pushes the posterior distribution of $m_{\beta}$ down to the region almost outside of the sensitivity of Project 8 in the NO case, it does not affect much the situation in the IO case due to the lower bound on $m_{\beta} \gtrsim 50 \mathrm{meV}$ even in the limit of $m_{3} \rightarrow 0$. This also implies that future tritium beta-decay experiments are able to discriminate between neutrino mass orderings.

As KATRIN continues to accumulate more beta-decay events and the development of the techniques to be deployed in Project 8 and PTOLEMY is well in progress, it is timely and necessary to revisit the effective neutrino mass and its validity in future beta-decay experiments. The analysis presented in the present work should be helpful in understanding the approximations made in expressions of the beta spectrum and is suggestive for the improvement on the usage of the effective masses. In light of the precision measurement of the beta spectrum already in the first run of 
KATRIN, one may go further to extend the analysis to consider the presence of sterile neutrinos and other new physics, and/or to consider the electron-capture decay of ${ }^{163} \mathrm{Ho}$.

\section{ACKNOWLEDGMENTS}

The authors would like to thank Yu-Feng $\mathrm{Li}$ and Prof. Zhi-zhong Xing for useful discussions. This work was supported in part by the National Natural Science Foundation of China under Grants No. 11775232 and No. 11835013 and by the CAS Center for Excellence in Particle Physics. W. R. is supported by the DFG with Grant No. RO 2516/7-1 in the Heisenberg program.

\section{APPENDIX A: EXPERIMENTAL SETUPS}

Some experimental details about KATRIN, Project 8 and PTOLEMY experiments are as follows:

(i) The KATRIN experiment [11] implements the so-called MAC-E-Filter (Magnetic Adiabatic Collimation combined with an Electrostatic Filter) to select electrons from tritium beta decays that can pass through the electrostatic barrier with the potential energy of $E_{\mathrm{V}}$. The observable in the MAC-EFilter is the integrated number of the electrons that have passed through the energy barrier. The sharpness of the filter is characterized by the ratio between the minimum $B_{\min }=3 \times 10^{-4} \mathrm{~T}$ and the maximum $B_{\max }=6 \mathrm{~T}$ of magnetic fields, i.e., $\Delta=$ $E_{e} B_{\min } / B_{\max } \approx 1 \mathrm{eV}$, where $E_{e} \approx Q=18.6 \mathrm{keV}$ is the electron energy in the range close to the end point and $Q \equiv m_{3^{3}}-m^{3} \mathrm{He}_{e}-m_{e}$ is the $Q$ value for tritium beta decay. Since the filter is insensitive to the transverse kinetic energy of electrons, the sharpness denotes roughly the maximum of transverse kinetic energies and thus can be regarded as the energy resolution. Adopting the energy window $E_{\mathrm{V}} \in[Q-30 \mathrm{eV}, Q+5 \mathrm{eV}]$ and including all the statistic and systematic uncertainties, the KATRIN experiment [11] with a target tritium mass of $\mathcal{O}\left(10^{-4}\right) \mathrm{g}$ can measure $m_{\beta}^{2}$ with a $1 \sigma$ uncertainty of $0.025 \mathrm{eV}^{2}$, corresponding to the sensitivity of $m_{\beta}<0.2 \mathrm{eV}$ at the $90 \% \mathrm{CL}$ in the assumption of $m_{\beta}=0$ as the true value. KATRIN can also directly measure the nonintegrated beta spectrum by extracting the time of flight information from the source to the detector, operating in the so-called MAC-E-TOF mode. Since the emitting time of the electron at source is not directly measurable, a technique has been devised to infer the emitting time by chopping the source with some high voltage potential frequently. A lower counting rate and a worse energy resolution will be caused by the additional chopping procedure. The total target mass of ${ }^{3} \mathrm{H}$ planned to be loaded in the full KATRIN setup can be inferred from the formula of the tritium molecule number $N\left(T_{2}\right)=A_{\mathrm{S}} \cdot \epsilon_{\mathrm{T}} \cdot \rho d \approx 2.518 \times$ $10^{19}$ with the source cross section $A_{\mathrm{S}}=53 \mathrm{~cm}^{2}$, the tritium purity $\epsilon_{\mathrm{T}}=0.95$, and the column density $\rho d=5 \times 10^{17} \mathrm{~cm}^{-2}$; see Eq. (25) and Table 7 of Ref. [11] for details. Given the mass per tritium nucleus $\sim 5 \times 10^{-24} \mathrm{~g}$, we obtain the total target mass of the full KATRIN as $m_{\text {KATRIN }}=2.5 \times 10^{-4} \mathrm{~g}$. The energy resolution of KATRIN in this work is fixed to $\Delta_{\text {KATRIN }}=1 \mathrm{eV}$.

(ii) Unlike the KATRIN experiment, the Project 8 Collaboration will utilize the technique of cyclotron radiation emission spectroscopy to measure the electron energies [17]. If the magnetic field is uniform in the spectrometer, the cyclotron radiation of accelerating electrons can be observed for a few microseconds and its frequency can be precisely determined, leading to an excellent energy resolution. As has already been shown in Fig. 5 of Ref. [17], with the deployment of $\mathcal{O}\left(10^{-4}\right) \mathrm{g}$ atomic ${ }^{3} \mathrm{H}$ and 1 year of running time, Project 8 is able to push the upper limit on the effective neutrino mass down to $m_{\beta}<40 \mathrm{meV}$ at the $90 \% \mathrm{CL}$, assuming the true value of $m_{\beta}=0$. In this work, we will adopt two extreme setups for Project 8: (i) an intermediate phase with the molecular ${ }^{3} \mathrm{H}$, a target mass of $m_{\mathrm{P} 8}=$ $5 \times 10^{-4} \mathrm{~g}$ corresponding to $5 \times 10^{19}$ tritium molecules, and an energy resolution $\Delta_{\mathrm{P} 8 \mathrm{~m}}=0.36 \mathrm{eV}$ limited by the irreducible width of the final state molecular excitations [35]; (ii) an ultimate phase with the atomic ${ }^{3} \mathrm{H}$, a target mass of $m_{\mathrm{P} 8}=5 \times 10^{-4} \mathrm{~g}$, and an energy resolution of $\Delta_{\mathrm{P} 8 \mathrm{a}}=0.05 \mathrm{eV}$ which is limited by the inhomogeneity of the magnetic field $\Delta B / B \sim 10^{-7}[17] .^{7}$ The target mass $m_{\mathrm{P} 8}=$ $5 \times 10^{-4} \mathrm{~g}$ can be achieved with a gas volume of $100 \mathrm{~m}^{2}$ as required by the phase IV of Project 8 and a gaseous tritium number density of $10^{12} \mathrm{~cm}^{-3}$.

(iii) The PTOLEMY experiment has been designed to detect the cosmic neutrino background $(\mathrm{C} \nu \mathrm{B})$ [18-20] via the electron-neutrino capture on tritium $\nu_{e}+{ }^{3} \mathrm{H} \rightarrow e^{-}+{ }^{3} \mathrm{He}$, as suggested by Steven Weinberg in 1962 [51]. Thanks to the large target mass of $100 \mathrm{~g}$ tritium and the low background rate required for the $\mathrm{C} \nu \mathrm{B}$ detection, PTOLEMY would have an overwhelmingly better sensitivity to the absolute neutrino mass than KATRIN does, namely, the relative uncertainty reaches $\sigma\left(m_{1}\right) / m_{1} \lesssim 10^{-2}$ for $m_{1}=10 \mathrm{meV}$ with an energy resolution of $\Delta=$ $100 \mathrm{meV}$. In the PTOLEMY experiment, the energy

\footnotetext{
${ }^{7}$ The energy resolution of Project 8 with atomic tritium may be roughly obtained by the relation $\Delta E / m_{e}=\Delta f / f \approx \Delta B / B$, where $f$ is the frequency of the cyclotron radiation and $B$ is the assumed nearly uniform magnetic field.
} 
of electrons from tritium beta decays will be measured in three steps. First, the MAC-E-Filter is used to select the electrons close to the end point, preventing the calorimeter from being swamped by the huge number of events in the energy range below the end point. Second, after passing through the MAC-E-Filter, the electrons are then sent to a long uniform solenoid, undergoing the cyclotron motion in the magnetic field of $2 \mathrm{~T}$. Hence, the radio signal can be implemented to track each single electron. Finally, the electrons are decelerated by the electrostatic voltage until their kinetic energies are $100 \mathrm{eV}$ or so to match the dynamic range of a cryogenic calorimeter. The energy resolution of these electrons can be as low as $50 \mathrm{meV}$ [20].

\section{APPENDIX B: EXPERIMENTAL LIKELIHOODS}

The likelihood functions from the following different classes of experiments have been used in our analysis: (i) oscillation experiments $\mathcal{L}_{\text {osc }}$; (ii) $\beta$-decay experiments $\mathcal{L}_{\beta}$; (iii) $0 \nu \beta \beta$ experiments $\mathcal{L}_{0 \nu \beta \beta}$; (iv) cosmological observations $\mathcal{L}_{\text {cosmo }}$. To be specific, we collect all the details of each likelihood function as follows:

(i) $\mathcal{L}_{\text {osc }}$-The likelihood information of neutrino oscillation experiments will be taken from the latest global-fit results of the Nu-Fit group [34]. The likelihood function can be obtained as $\mathcal{L}_{\text {osc }}=$ $\exp \left(-\Delta \chi^{2} / 2\right)$ with $\Delta \chi^{2}$ defined as

$$
\Delta \chi^{2} \equiv \sum_{i} \frac{\left(\Theta_{i}-\Theta_{i}^{\mathrm{bf}}\right)^{2}}{\sigma_{i}^{2}},
$$

where $\Theta_{i} \in\left\{\sin ^{2} \theta_{13}, \sin ^{2} \theta_{12}, \Delta m_{\text {sol }}^{2}, \Delta m_{\text {atm }}^{2}\right\}, \Theta_{i}^{\text {bf }}$ is the best-fit value of the parameter from the global analysis, and $\sigma_{i}$ is the symmetrized $1 \sigma$ error. We take the following central values and symmetrized $1 \sigma$ errors of oscillation parameters relevant for the $\beta$ decays:

$$
\begin{aligned}
\sin ^{2} \theta_{12} & =(3.10 \pm 0.12) \times 10^{-1}, \\
\Delta m_{\mathrm{sol}}^{2} & =(7.39 \pm 0.20) \times 10^{-5} \mathrm{eV}^{2}, \\
\sin ^{2} \theta_{13} & =(2.241 \pm 0.065) \times 10^{-2}, \\
\Delta m_{\mathrm{atm}}^{2} & =(2.525 \pm 0.032) \times 10^{-3} \mathrm{eV}^{2}
\end{aligned}
$$

for $\mathrm{NO}$, and

$$
\begin{aligned}
\sin ^{2} \theta_{12} & =(3.10 \pm 0.12) \times 10^{-1}, \\
\Delta m_{\text {sol }}^{2} & =(7.39 \pm 0.20) \times 10^{-5} \mathrm{eV}^{2}, \\
\sin ^{2} \theta_{13} & =(2.264 \pm 0.066) \times 10^{-2}, \\
\Delta m_{\text {atm }}^{2} & =(-2.512 \pm 0.033) \times 10^{-3} \mathrm{eV}^{2}
\end{aligned}
$$

for IO. The preference of NO over IO can be represented by the difference of their $\chi^{2}$ minima, i.e., $\Delta \chi_{\mathrm{MO}}^{2}=\chi_{\mathrm{NO}}^{2}-\chi_{\mathrm{IO}}^{2} \simeq 9.3$, implying a more than $3 \sigma$ preference of $\mathrm{NO}$.

(ii) $\mathcal{L}_{\beta}$ - By measuring the end point of the $\beta$ decay spectrum, the tritium $\beta$-decay experiments (e.g., Troitsk [13], Mainz [12], and KATRIN [9,10]) can already provide us good constraints on the absolute neutrino mass scale via the effective neutrino mass $m_{\beta} \equiv\left(\sum_{i} m_{i}^{2}\left|U_{e i}\right|^{2}\right)^{1 / 2}$. The limits of the former two are given as

$$
\begin{aligned}
& m_{\beta}^{2}=-0.67 \pm 2.53 \mathrm{eV}^{2} \text { (Troitsk) } \\
& m_{\beta}^{2}=-0.6 \pm 3.0 \mathrm{eV}^{2} \text { (Mainz) }
\end{aligned}
$$

Similar to Eq. (B1) in the case of neutrino oscillations, the likelihood function can be constructed with the central values and $1 \sigma$ errors of $m_{\beta}^{2}$ in Eq. (B2). For KATRIN, we use the likelihood presented in Fig. 4 of Ref. [9]. We find the likelihood can be well approximated by a skewed normal distribution

$$
\begin{aligned}
\mathcal{L}_{\text {KATRIN }}\left(m_{\beta}^{2}\right) \propto & \frac{1}{\sqrt{2 \pi} \sigma} \exp \left[-\frac{\left(m_{\beta}^{2}-\mu\right)^{2}}{2 \sigma^{2}}\right] \\
& \times \operatorname{erfc}\left[-\frac{\alpha\left(m_{\beta}^{2}-\mu\right)}{\sqrt{2} \sigma}\right],
\end{aligned}
$$

where $\operatorname{erfc}(x)$ is the complementary error function, with $\sigma=1.506 \mathrm{eV}^{2}, \mu=0.0162 \mathrm{eV}^{2}$, and $m_{\beta}^{2}$ in units of $\mathrm{eV}^{2}$, as well as $\alpha=-2.005$. Since the KATRIN experiment has the highest sensitivity to $m_{\beta}$, we may have $\mathcal{L}_{\beta} \approx \mathcal{L}_{\text {KATRIN }}$.

(iii) $\mathcal{L}_{0 \nu \beta \beta}$-The constraints on the half-life of $0 \nu \beta \beta$ are given by the existing $0 \nu \beta \beta$ searches. The limits on the effective neutrino mass $\left|m_{\beta \beta}\right|$ can be derived by using

$$
\left(T_{1 / 2}^{0 \nu}\right)^{-1}=G_{0 \nu}\left|\mathcal{M}_{0 \nu}\right|^{2} \frac{\left|m_{\beta \beta}\right|^{2}}{m_{e}^{2}},
$$

where $G_{0 \nu}$ denotes the phase-space factor, $\mathcal{M}_{0 \nu}$ is the nuclear matrix element (NME), and $m_{e}=$ $0.511 \mathrm{MeV}$ is the electron mass. In our numerical analysis, we use the likelihood functions from Refs. [39,52], which include the experimental information of GERDA [53], KamLAND-Zen [54], EXO [55], and CUORE [52].

(iv) $\mathcal{L}_{\text {cosmo }}$-The cosmological observations can set very strong constraints on the sum of the three neutrino masses $\Sigma \equiv m_{1}+m_{2}+m_{3}$. The Planck Collaboration has recently updated their results in Ref. [56]. For illustration, we will adopt the 
likelihood functions by combining different datasets which yield the following bounds on the sum of the three neutrino masses at the $95 \% \mathrm{CL}$ :

$$
\begin{aligned}
& \Sigma<0.54 \mathrm{eV}\left(\mathcal{L}_{\text {cosmo }}^{(1)}, \text { Planck TT + lowE }\right), \\
& \Sigma<0.24 \mathrm{eV}\left(\mathcal{L}_{\text {cosmo }}^{(2)}, \text { Planck TT, TE, EE + lowE + lensing }\right), \\
& \Sigma<0.12 \mathrm{eV}\left(\mathcal{L}_{\text {cosmo }}^{(3)}, \text { Planck TT, TE, EE + lowE + lensing }+ \text { BAO }\right) .
\end{aligned}
$$

The likelihood functions have been obtained by analyzing the Markov chain files available from the Planck Legacy Archive.

With the likelihood functions listed above, the total likelihood relevant for our analysis can be calculated as $\mathcal{L}_{\text {tot }}=$ $\mathcal{L}_{\text {osc }} \times \mathcal{L}_{\beta} \times \mathcal{L}_{0 \nu \beta \beta} \times \mathcal{L}_{\text {cosmo }}^{(i)}($ for $i=1,2,3$ ).

[1] E. W. Otten and C. Weinheimer, Neutrino mass limit from tritium beta decay, Rep. Prog. Phys. 71, 086201 (2008).

[2] M. J. Dolinski, A. W. P. Poon, and W. Rodejohann, Neutrinoless double-beta decay: Status and prospects, Annu. Rev. Nucl. Part. Sci. 69, 219 (2019).

[3] M. Lattanzi and M. Gerbino, Status of neutrino properties and future prospects-Cosmological and astrophysical constraints, Front. Phys. 5, 70 (2018).

[4] E. Fermi, An attempt of a theory of beta radiation. 1., Z. Phys. 88, 161 (1934).

[5] E. Fermi, Trends to a theory of beta radiation. (In Italian), Nuovo Cimento 11, 1 (1934).

[6] F. Perrin, Possibility of emission of neutral particles with zero intrinsic mass in beta radioactivity. (In French), Comptes Rendus 197, 1625 (1933).

[7] R. E. Shrock, New tests for, and bounds on, neutrino masses and lepton mixing, Phys. Lett. 96B, 159 (1980).

[8] M. Tanabashi et al. (Particle Data Group), Review of particle physics, Phys. Rev. D 98, 030001 (2018).

[9] M. Aker et al. (KATRIN Collaboration), An Improved Upper Limit on the Neutrino Mass from a Direct Kinematic Method by KATRIN, Phys. Rev. Lett. 123, 221802 (2019).

[10] M. Aker et al. (KATRIN Collaboration), First operation of the KATRIN experiment with tritium, arXiv:1909.06069.

[11] J. Angrik et al. (KATRIN Collaboration), KATRIN design report 2004, https://www.katrin.kit.edu/publikationen/ DesignReport2004-12Jan2005.pdf.

[12] C. Kraus et al., Final results from phase II of the Mainz neutrino mass search in tritium beta decay, Eur. Phys. J. C 40, 447 (2005).

[13] V. N. Aseev et al. (Troitsk Collaboration), An upper limit on electron antineutrino mass from Troitsk experiment, Phys. Rev. D 84, 112003 (2011).

[14] L. Gastaldo et al., The electron capture in ${ }^{163} \mathrm{Ho}$ experiment-ECHo, Eur. Phys. J. ST 226, 1623 (2017).

[15] B. Alpert et al., HOLMES-the electron capture decay of ${ }^{163} \mathrm{Ho}$ to measure the electron neutrino mass with sub-eV sensitivity, Eur. Phys. J. C 75, 112 (2015).

[16] J. W. Engle, E. R. Birnbaum, H. R. Trellue, K. D. John, M. W. Rabin, and F.M. Nortier, Evaluation of ${ }^{163} \mathrm{Ho}$ production options for neutrino mass measurements with microcalorimeter detectors, Nucl. Instrum. Methods B 311, 131 (2013).

[17] A. Ashtari Esfahani et al. (Project 8 Collaboration), Determining the neutrino mass with cyclotron radiation emission spectroscopy-Project 8, J. Phys. G 44, 054004 (2017).

[18] S. Betts et al., Development of a relic neutrino detection experiment at PTOLEMY: Princeton tritium observatory for light, early-universe, massive-neutrino yield, in Proceedings, 2013 Community Summer Study on the Future of U.S. Particle Physics: Snowmass on the Mississippi (CSS2013): Minneapolis, MN, USA, 2013 (2013), https://www.slac .stanford.edu/econf/C1307292/docs/submittedArxivFiles/ 1307.4738.pdf.

[19] E. Baracchini et al. (PTOLEMY Collaboration), PTOLEMY: A proposal for thermal relic detection of massive neutrinos and directional detection of $\mathrm{MeV}$ dark matter, arXiv: 1808.01892 .

[20] M. G. Betti et al. (PTOLEMY Collaboration), Neutrino physics with the PTOLEMY project: Active neutrino properties and the light sterile case, J. Cosmol. Astropart. Phys. 07 (2019) 047.

[21] S. S. Masood, S. Nasri, J. Schechter, M. A. Tortola, J. W. F. Valle, and C. Weinheimer, Exact relativistic beta decay endpoint spectrum, Phys. Rev. C 76, 045501 (2007).

[22] F. Simkovic, R. Dvornicky, and A. Faessler, Exact relativistic tritium beta-decay endpoint spectrum in a hadron model, Phys. Rev. C 77, 055502 (2008).

[23] A. J. Long, C. Lunardini, and E. Sabancilar, Detecting nonrelativistic cosmic neutrinos by capture on tritium: Phenomenology and physics potential, J. Cosmol. Astropart. Phys. 08 (2014) 038.

[24] P. O. Ludl and W. Rodejohann, Direct neutrino mass experiments and exotic charged current interactions, J. High Energy Phys. 06 (2016) 040.

[25] F. Vissani, Nonoscillation searches of neutrino mass in the age of oscillations, Nucl. Phys. B, Proc. Suppl. 100, 273 (2001).

[26] A. Saenz, S. Jonsell, and P. Froelich, Improved Molecular Final-State Distribution of $\mathrm{HeT}+$ for the $\beta$-Decay Process of T2, Phys. Rev. Lett. 84, 242 (2000). 
[27] O. Fackler, B. Jeziorski, W. Kolos, H. J. Monkhorst, and K. Szalewicz, Accurate Theoretical Beta Decay Energy Spectrum of the Tritium Molecule and its Neutrino Mass Dependence, Phys. Rev. Lett. 55, 1388 (1985).

[28] N. Doss, J. Tennyson, A. Saenz, and S. Jonsell, Molecular effects in investigations of tritium molecule beta decay endpoint experiments, Phys. Rev. C 73, 025502 (2006).

[29] L. I. Bodine, D. S. Parno, and R. G. H. Robertson, Assessment of molecular effects on neutrino mass measurements from tritium $\beta$ decay, Phys. Rev. C 91, 035505 (2015).

[30] Y. Farzan, O. L. G. Peres, and A. Yu. Smirnov, Neutrino mass spectrum and future beta decay experiments, Nucl. Phys. B612, 59 (2001).

[31] J. Studnik and M. Zralek, Parametrization of the energy spectrum in the tritium beta decay, arXiv:hep-ph/0110232.

[32] Y. Farzan and A. Yu. Smirnov, On the effective mass of the electron neutrino in beta decay, Phys. Lett. B 557, 224 (2003).

[33] J. F. Beacom and N. F. Bell, Do solar neutrinos decay?, Phys. Rev. D 65, 113009 (2002).

[34] I. Esteban, M. C. Gonzalez-Garcia, A. HernandezCabezudo, M. Maltoni, and T. Schwetz, Global analysis of three-flavour neutrino oscillations: Synergies and tensions in the determination of $\theta_{23}, \delta_{C P}$, and the mass ordering, J. High Energy Phys. 01 (2019) 106.

[35] B. Monreal and J. A. Formaggio, Relativistic cyclotron radiation detection of tritium decay electrons as a new technique for measuring the neutrino mass, Phys. Rev. D 80, 051301 (2009).

[36] R. Trotta, Bayes in the sky: Bayesian inference and model selection in cosmology, Contemp. Phys. 49, 71 (2008).

[37] G. Benato, Effective majorana mass and neutrinoless double beta decay, Eur. Phys. J. C 75, 563 (2015).

[38] A. Di Iura and D. Meloni, Probability densities of the effective neutrino masses $m_{-} \beta$ and $m_{-} \beta \beta$, Nucl. Phys. B921, 829 (2017).

[39] A. Caldwell, A. Merle, O. Schulz, and M. Totzauer, Global Bayesian analysis of neutrino mass data, Phys. Rev. D 96, 073001 (2017).

[40] S.-F. Ge, W. Rodejohann, and K. Zuber, Half-life expectations for neutrinoless double beta decay in standard and non-standard scenarios, Phys. Rev. D 96, 055019 (2017).

[41] M. Agostini, G. Benato, and J. Detwiler, Discovery probability of next-generation neutrinoless double- $\beta$ decay experiments, Phys. Rev. D 96, 053001 (2017).
[42] G.-Y. Huang and S. Zhou, Impact of an eV-mass sterile neutrino on the neutrinoless double-beta decays: A Bayesian analysis, Nucl. Phys. B945, 114691 (2019).

[43] S. Dell'Oro, S. Marcocci, and F. Vissani, Empirical inference on the Majorana mass of the ordinary neutrinos, Phys. Rev. D 100, 073003 (2019).

[44] Z.-z. Xing, Flavor structures of charged fermions and massive neutrinos, arXiv:1909.09610.

[45] S. Gariazzo, Constraining power of open likelihoods, made prior-independent, arXiv:1910.06646.

[46] J. Kotila and F. Iachello, Phase space factors for double- $\beta$ decay, Phys. Rev. C 85, 034316 (2012).

[47] F. Feroz and M. P. Hobson, Multimodal nested sampling: An efficient and robust alternative to MCMC methods for astronomical data analysis, Mon. Not. R. Astron. Soc. 384, 449 (2008).

[48] F. Feroz, M. P. Hobson, and M. Bridges, MultiNest: An efficient and robust Bayesian inference tool for cosmology and particle physics, Mon. Not. R. Astron. Soc. 398, 1601 (2009).

[49] F. Feroz, M. P. Hobson, E. Cameron, and A. N. Pettitt, Importance nested sampling and the multinest algorithm, arXiv:1306.2144.

[50] S. M. Bilenky, M. D. Mateev, and S. T. Petcov, A comment on the measurement of neutrino masses in beta-decay experiments, Phys. Lett. B 639, 312 (2006).

[51] S. Weinberg, UnCiversal neutrino degeneracy, Phys. Rev. 128, 1457 (1962).

[52] C. Alduino et al. (CUORE Collaboration), First Results from CUORE: A Search for Lepton Number Violation via $0 \nu \beta \beta$ Decay of ${ }^{130}$ Te, Phys. Rev. Lett. 120, 132501 (2018).

[53] M. Agostini et al., Background-free search for neutrinoless double- $\beta$ decay of ${ }^{76} \mathrm{Ge}$ with GERDA, Nature (London) 544, 47 (2017).

[54] A. Gando et al. (KamLAND-Zen Collaboration), Search for Majorana Neutrinos near the Inverted Mass Hierarchy Region with KamLAND-Zen, Phys. Rev. Lett. 117, 082503 (2016); Addendum, Phys. Rev. Lett.117, 109903 (2016).

[55] J. B. Albert et al. (EXO-200 Collaboration), Search for Majorana neutrinos with the first two years of EXO-200 data, Nature (London) 510, 229 (2014).

[56] N. Aghanim et al. (Planck Collaboration), Planck 2018 results. VI. Cosmological parameters, arXiv:1807.06209. 